\title{
An Effective Model of Endogenous Clocks and External Stimuli Determining Ciradian Rhythms
}

\section{Tim Breitenbach}

University of Würzburg

Charlotte Helfrich-Förster

University of Würzburg

Thomas Dandekar ( $\nabla$ dandekar@biozentrum.uni-wuerzburg.de )

University of Würzburg

\section{Research Article}

Keywords: circadian clock, external stimuli, couple models, entrainment, relative coordination, phase response curve, permanent stimulus, enzyme kinetics, modeling of biological systems

Posted Date: March 12th, 2021

DOl: https://doi.org/10.21203/rs.3.rs-273637/v1

License: @ (i) This work is licensed under a Creative Commons Attribution 4.0 International License.

Read Full License

Version of Record: A version of this preprint was published at Scientific Reports on August 9th, 2021. See the published version at https://doi.org/10.1038/s41598-021-95391-y. 


\title{
An effective model of endogenous clocks and external stimuli determining circadian rhythms
}

\author{
Tim Breitenbach* Charlotte Helfrich-Förster ${ }^{\dagger}$ Thomas Dandekar ${ }^{\ddagger}$
}

\begin{abstract}
Circadian endogenous clocks of eukaryotic organisms are an established and rapidly developing research field. To investigate and simulate in an effective model the effect of external stimuli on such clocks and their components we developed a software framework for download and simulation. The application is useful to understand the different involved effects in a mathematical simple and effective model. This concerns the effects of Zeitgebers, feedback loops and further modifying components.

We start from a known mathematical oscillator model, which is based on experimental molecular findings. This is extended with an effective framework that includes the impact of external stimuli on the circadian oscillations including high dose pharmacological treatment. The external stimuli framework defines a systematic procedure by input-output-interfaces to couple different oscillators. With a similar idea the issue of singularity points of circadian clocks is modeled and related to different model approaches. Tables explain modifications for specific organisms and by different modules. The mathematical framework and formalism is generic and allows to study in general the effect of external stimuli on oscillators and other biological processes.

For an easy replication of each numerical experiment presented in this work and an easy implementation of the framework the corresponding Mathematica files are fully made available. They can be downloaded at the following link: https://www.biozentrum.uniwuerzburg.de/bioinfo/computing/circadian/.
\end{abstract}

Keywords circadian clock, external stimuli, couple models, entrainment, relative coordination, phase response curve, permanent stimulus, enzyme kinetics, modeling of biological systems

\section{Introduction}

Circadian clocks are daily time-keeping mechanisms that help organisms to anticipate the regular 24-hour fluctuation on earth. The rhythms generated by circadian clocks are of endogenous nature and persist even in the absence of environmental cues with a species-specific period that usually deviates from 24 hours. They are synchronized (=entrained) to the 24-hour rhythm on earth

\footnotetext{
*Institut für Mathematik, Universität Würzburg, Emil-Fischer-Strasse 30, 97074 Würzburg, Germany; tim.breitenbach@mathematik.uni-wuerzburg.de

${ }^{\dagger}$ Biozentrum, Universität Würzburg, Am Hubland, 97074 Würzburg, Germany; charlotte.foerster@biozentrum.uni-wuerzburg.de

†Biozentrum, Universität Würzburg, Am Hubland, 97074 Würzburg, Germany; dandekar@biozentrum.uniwuerzburg.de
} 
by several environmental cues, called Zeitgebers (German for "time-givers", "synchronizers"), of which light has the largest impact. As nicely summarized in [1], circadian clocks have the following well defined properties: (1) they entrain to Zeitgeber cycles with a limited range of entrainment, (2) they can follow phase shifts of Zeitgeber cycles, but they need a certain number of transient cycles until they have established their previous phase to the Zeitgeber cycle, (3) they can be phase-shifted by light-pulses in a time-dependent manner that is characterized in a phase-response curve, (4) they free-run under constant conditions with a species-specific period, which is lightdependent. Constant light either lengthens or shortens the free-running period and constant light of high intensity dampens the clock and finally leads to arrhythmicity. Our aim is to start from a known mathematical oscillator model based on experimental findings and extend it to an effective modeling framework for the circadian clock that includes the impact of external stimuli.

Although the general properties of circadian clocks are the same in all organisms, species-specific differences exist. When comparing for example the mouse circadian clock with the fruit fly circadian clock, which are standard models for mammalian and insect clocks, the following differences become evident: (1) The mouse (Mus musculus) clock has a narrower range of entrainment and it needs longer to follow phase-shifts of the Zeitgeber cycle than the fruit fly (Drosophila melanogaster) clock. This suggests that it is less plastic and less light-sensitive. (2) In line with this suggestion, the mouse phase-response curve to light has a lower amplitude than that of the fruit fly and the mouse free-running period is less responsive to the light intensity. Our framework presents an effective model covering all these variants of a circadian clock.

Some of these differences can be explained on the molecular level. In both model systems, circadian rhythms are generated by molecular negative feedback loops in which the gene products of certain clock genes inhibit their own transcriptional activators after a time delay (reviewed in [2]; [3]). The orthologous transcriptional activators are called CLOCK and BMAL1 in Mus and CLOCK and CYCLE in Drosophila. They form heterodimers that activate transcription of the genes cryptochrome 1 and 2 (cry1/2) and period 1 and $2($ per1/2) in Mus and period (per) and timeless (tim) in Drosophila. PER1/2-mCRY1/2 protein complexes in Mus and PER-TIM protein complexes in Drosophila are modified (e.g. phosphorylated) and after a time delay inactivate the CLOCK-BMAL1 and CLK-CYC activators, respectively, to repress transcription. PER1/2CRY1/2 and PER-TIM complexes are subsequently degraded, which permits the activators to initiate the next cycle of transcription. We solve this in one framework by adding corresponding terms to existing clock models denoted in Table 1.

A main difference exists in the effects of light on this negative feedback loop: whereas light induces the transcription of per $1 / 2$ in the mouse, light leads to the degradation of TIM in the fruit fly (reviewed in [2]). The higher light sensitivity of the fruit fly clock can be explained by the presence of a light-sensitive form of Cryptochrome (Drosophila CRY) that is not part of the negative feedback loop but instead interacts with TIM in the presence of light ([4]; [5]). Drosophila CRY is expressed in the clock neurons and leads to an immediate degradation of TIM and a reset of the clock in the presence of light [6]. This is true for all tissues in which the clock is ticking, not only the master clock in the brain [7]. Thus, the master clock in the brain and the body clocks run with the same phase and both are quickly entrainable by light. In contrast, mouse CRYs are light-insensitive and the information about light has to reach the master clock in the brain, specifically the suprachiasmatic nucleus (SCN), via the eyes and the retinohypothalamic tract (reviewed by [8]). The mouse master clock in the SCN then synchronizes the clocks in the body. Thus, there are several steps between light reception and entrainment of the master clock as well as between entrainment of the master clock and the body clocks, also called peripheral oscillators. As a consequence the peripheral oscillators are phase-delayed by $\sim 6$ hours in respect 
to the master clock [9]. The peripheral oscillators are not only entrainable by the master clock, but in addition by other Zeitgebers like feeding ([10]). The main focus of the presented work is to show how to systematically generate sub models and combine them to a total model demonstrated by coupling these different oscillators.

More specific, the aim of the present paper is hence to investigate a purposeful framework to include external stimuli into a model and thus generate a systematic way to couple models with an input-output-interface. We derive an effective model and demonstrate the usefulness with a simple mathematical model of the circadian clock since the circadian clock is affected by many external stimuli. Moreover, as mentioned, the inner clock of an organism consists of many coupled oscillators which is a clear use case for an input-output-interface. We use only the minimal components necessary to reproduce the basic observed model behavior (see experiments below) to keep the discussion clear and focused to the discussion of the input-output-framework with external stimuli. Our model starts from the formalism and original model of Goldbeter (1995), [11] and is extended with an input-output-framework with external stimuli. We show and focus on the properties and interactions in this system which are critical to reproduce, study and analyze important system behavior of circadian clocks as summarized in Table 1.

In our work, we extend this model with additional terms modeling specific effects from different origin. Table 1 gives an overview over the simulated experiments and where the data can be found in the present work. We remark that we have to be careful with external stimuli that change reaction parameters of the model [11] noteworthy, like temperature or certain chemical agents, since the model of Goldbeter is based on enzyme kinetics where model parameters, like reaction coefficients, depend on temperature for example. These parameters are assumed to be constants in this work for the scope of the paper which is to discuss the input-output-interface of models. However it paves the way for extension by letting the "constants" depend on temperature or on other chemicals like ions in case a more detailed model is needed. The effect of temperature and chemicals can also be modeled by including corresponding enzyme kinetic terms into the model instead of changing model parameters where the effect of the temperature or chemicals act as the external stimuli.

In the present work we focus on establishing a general framework for the effective inclusion of external stimuli. That means how to include only necessary information of the effect of external stimuli on the agents of a biological system to be able to explain the effect of external stimuli on behavior sufficiently well. This framework has already been used to extend SQUAD models for regulatory networks $[12,13]$. Now, we demonstrate the wide applicability of the proposed framework, in particular to models for enzyme kinetics, exemplary based on the model of Goldbeter [11], which is without temperature dependency of the parameters, by reproducing many experimental outcomes with a single external stimuli framework, see Table 1. This works because a lot of experiments are done with light, where temperature is constant during the experiments or chemicals used do not change the reaction parameters of the enzymes for the molecular clock.

The focus of this work is not on calculating all specific effects in Table 1 in detail but introducing a framework and a systematic procedure that is able to include all these effects and simulate them. An advantage of our framework of including external stimuli is that it is independent of the model chosen. There is a considerable body of literature making the biological model of the circadian clock more complete, for instance studying the specific components present in the fly or mammalian circadian clock (see Table 2). The simulation is then easily extended including these proper formulas in our Mathematica framework. For example, the ideas presented in this work how to include external stimuli into circadian clock models can easily be extended to more complex models of the fly model shown in [23]. With analogous terms as presented in this work 


\begin{tabular}{|c|c|c|}
\hline experiment & exp. evidence & descr. in present work \\
\hline \hline synchronization to external Zeitgeber & {$[14]$} & Figure 2a, 2c, 2e, Sup. Fig. 4 \\
\hline restart of the endogenous clock & {$[15]$} & Supplementary Figure 3 \\
\hline Entrainment to different external periods & {$[16]$} & paragraph following $(21)$ \\
\hline permanent stimulus of constant intensity & {$[17,18]$} & Figure 4e \\
\hline phase-response curves & {$[19,20,21]$} & Spplementary Figure 7e \\
\hline synchronization of peripheral clocks by the SCN & {$[22,10]$} & Figure 5a, 5c, 5e, 6 \\
\hline
\end{tabular}

Table 1: The table summarizes experiments with corresponding literature and where it is covered in the present work.

\begin{tabular}{|c|c|c|}
\hline Literature & Model equations & description \\
\hline \hline$[31]$ & $(1)-(2)$ & basic oscillations \\
\hline$[26]$ & $(2),(3),(4)$ & intercommunication \\
\hline$[32]$ & Table 2 & mathematical oscillator models \\
\hline$[23]$ & $(1 \mathrm{a})-(1 \mathrm{j})$ & PER/TIM complex \\
\hline$[29]$ & supporting text & mammalian circadian clock \\
\hline$[30]$ & Appendix 1 & detailed mammalian model \\
\hline
\end{tabular}

Table 2: In this table there is a selection of model equations that can be chosen for the combination with our external stimuli framework instead of the Goldbeter model used in this work by substituting it by the equations referenced in the column "Model equations".

where we let light act on PER we can model the effect of light on the expression of TIM. The terms which model the external stimuli perform their effect by increasing or decreasing the level of expression of the corresponding gene. The same works for models that are for mammalians. There are models ranging from basic $[24,25,26,27]$ to complex $[28,29,30]$ where in particular PER and CRY interactions are modeled. Also in this case the corresponding equations that model the expression of, e.g., PER and CRY are supplemented by the same terms that are presented in this work in order to influence their expression by the action of the modeled stimuli. Once the external stimuli are included, we can analyze the model with respect to an optimal interaction with the external stimuli as shown in $[12,13]$ in order to influence the circadian clock and thus the cell behavior. This is in particularly attractive if we think of external stimuli as drugs that interact with the circadian clock in order to calculate optimal therapies for which our proposed method how to include external stimuli can also be used.

However, to derive such a more complex and detailed model of the circadian clock is not the aim of the present work. The aim is to establish a systematic input-output-interface demonstrated on the topic of circadian clock. This formalism has a systematic bilinear structure where the function of the external stimuli is multiplied by another quantity. According to the law of mass action our presented formalism is a building block for more complex situations and thus we present a rational approach to model biological systems with as many equations as necessary to capture effects like cooperativity or until the model cannot be rejected based on experimental data. By the possibility of considering external stimuli as input functions for models, we show how to couple different models by replacing the external stimuli by the corresponding output functions of another model, as presented in Section 6 and Section 7. In this way, we can test and refine each single (sub) model for small biological systems and consequently have building blocks for constructing 
big and thus complex biological models by well validated small models. This usually makes the procedure of modeling more efficient since the parts of the models that possibly need to be refined can be identified fast and adjusted for the corresponding situation. For this purpose, the external stimuli of a sub model and its output need to be measured and validated if the simulated output fits sufficiently well to the measured output, which is the experimental data.

In order to describe the circadian clock modeling framework, we start from the basic model of Goldbeter which we introduce in the following. Subsequently, we give an introducing example entraining the model to light dark cycles. After discussing some modeling issues to adapt the model to different species, we show results oft the model under constant light and validate these results with experimental results from literature. Then we show how to combine these basic models to generate a total model of synchronizing different clocks in an organism. In the Supplementary material, we present additional basic experiments of a single oscillator/model that we found in the literature to further validate our external stimuli framework by showing that we obtain the same results. Furthermore, the examples of the Supplementary material demonstrate how to model different effects with our external stimuli framework to become more familiar with the framework. In the Discussion, we relate our work to existing publications and give an outlook for future work by discussing an explanation of singularity points (one pulse of light can stop the oscillation and another can start the oscillation again) from our model.

For an easy replication of each numerical experiment presented in this work and an easy implementation of the framework the corresponding Mathematica files are fully made available. They can be downloaded at the following link:

https://www.biozentrum.uni-wuerzburg.de/bioinfo/computing/circadian/.

\section{Results}

We recall the basic model of Goldbeter before we demonstrate how to couple different oscillators.

\section{The model of Goldbeter}

In the work of [11], a model based on enzyme kinetics and ordinary differential equations is described that provides limit cycles and thus can be used to model endogenous oscillations of proteins in a cell. Specifically, that model describes the oscillations of the Drosophila period protein (PER) and its multiple phosphorylation. A schematic of the network and a detailed description is given in Figure 1a and the corresponding system of ordinary differential equations is given by

$$
\begin{aligned}
\frac{d}{d t} M & =v_{s} \frac{K_{1}^{n}}{K_{I}^{n}+P_{N}^{n}}-v_{m} \frac{M}{K_{m}+M} \\
\frac{d}{d t} P_{0} & =k_{s} M-V_{1} \frac{P_{0}}{K_{1}+P_{0}}+V_{2} \frac{P_{1}}{K_{2}+P_{1}} \\
\frac{d}{d t} P_{1} & =V_{1} \frac{P_{0}}{K_{1}+P_{0}}-V_{2} \frac{P_{1}}{K_{2}+P_{1}}-V_{3} \frac{P_{1}}{K_{3}+P_{1}}+V_{4} \frac{P_{2}}{K_{4}+P_{2}} \\
\frac{d}{d t} P_{2} & =V_{3} \frac{P_{1}}{K_{3}+P_{1}}-V_{4} \frac{P_{2}}{K_{4}+P_{2}}-k_{1} P_{2}+k_{2} P_{N}-v_{d} \frac{P_{2}}{K_{d}+P_{2}} \\
\frac{d}{d t} P_{N} & =k_{1} P_{2}-k_{2} P_{N}
\end{aligned}
$$


where $M$ is the concentration of per mRNA in the cytosol, $P_{0}$ is the concentration of unphosphorylated, $P_{1}$ the concentration of monophosphorylated, $P_{2}$ the concentration of biphosphorylated PER protein, $P_{N}$ is the concentration of the biphosphorylated PER protein in the nucleus. Concentrations are defined with respect to the total cell volume.

Also in the work of [11], the model used in the present work is validated with experimental results. For our calculation with the model (1) to (5) we use the following values for the parameters which can be found in [11, Figure 2]. We have $v_{s}=0.76 \frac{\mu \mathrm{M}}{h}, v_{m}=0.65 \frac{\mu \mathrm{M}}{h}, K_{m}=0.5 \mu \mathrm{M}$, $k_{s}=0.38 \frac{1}{h}, v_{d}=0.95 \frac{\mu \mathrm{M}}{h}, k_{1}=1.9 \frac{1}{h}, k_{2}=1.3 \frac{1}{h}, K_{I}=1 \mu \mathrm{M}, K_{d}=0.2 \mu \mathrm{M}, n=4, K_{1}=K_{2}=K_{3}=$ $K_{4}=2 \mu \mathrm{M}, V_{1}=3.2 \frac{\mu \mathrm{M}}{h}, V_{2}=1.58 \frac{\mu \mathrm{M}}{h}, V_{3}=5 \frac{\mu \mathrm{M}}{h}, V_{4}=2.5 \frac{\mu \mathrm{M}}{h}$ with the initial values $M(0)=0.5$, $P_{0}(0)=0.5, P_{1}(0)=0.5, P_{2}(0)=0.6, P_{N}(0)=1.5$. We always calculate the model from time $t=0$ to a final time. The calculations in this work are performed with Wolfram Mathematica. In Figure 1b, we have a plot of the model where we see an almost 24 hours rhythm analogous to [11, Figure 2]. Throughout the work the time is plotted on the abscissa and the corresponding concentration with respect to the whole cell volume is plotted on the ordinate if not otherwise stated.

In the following, we introduce a systematic procedure that results in effective models that include the interaction of external stimuli with a studied biological system. For the demonstration of the practicability of this procedure we give several examples including reproduction of known results from different models and new model possibilities that are provided by our approach of considering external stimuli. For this purpose, we include bilinear control terms in order to alter the corresponding state quantities of the core model.

\section{Entrainment to light-dark cycles}

We extend the model of [11] by a mechanism that allows to include external stimuli. We know that external stimuli influence the circadian clock and can synchronize the rhythm to the external time phase. Such an external stimulus is for example light, see [14] or any other substances that influence the concentration of an agent of the model (1) to (5). An important application aspect is that tolerance to harsh pharmacological treatment such as bone marrow transplantation, radiation or mitigation of graft versus host disease depends on the time of the day. Knowing the influence of external stimuli on the circadian clocks is hence important for any pharmacological treatment, in particular at high dose treatment regimes, but is usually not attempted as a general framework for modeling such influences is lacking. This is, however, provided here.

In our first experiment, we synchronize the phase of the endogenous clock to the phase of an external Zeitgeber. We start our discussion by introducing now external influences changing the circadian oscillator. A simple influence assumes that the external stimulus leads to a further decay of per mRNA (M) (e.g. achieved by expressing a miRNA in the system or a treatment via any suitable drug). Since this is an effective model, we can just as well model the effect on the protein concentration with a similar effect. We model the further decay of per mRNA by

$$
\frac{d}{d t} M=v_{s} \frac{K_{1}^{n}}{K_{1}^{n}+P_{N}^{n}}-v_{m} \frac{M}{K_{m}+M}-\alpha u M
$$

instead of (1). The term $-u M$ models the effect that if the external stimulus is strong and $M$ is big, then the decay of $M$ is strong, that means a lot of per mRNA is degraded and if the external

stimulus is weak and $M$ is low, then there is almost no additional decay of $M$. The constant $\alpha$ is 

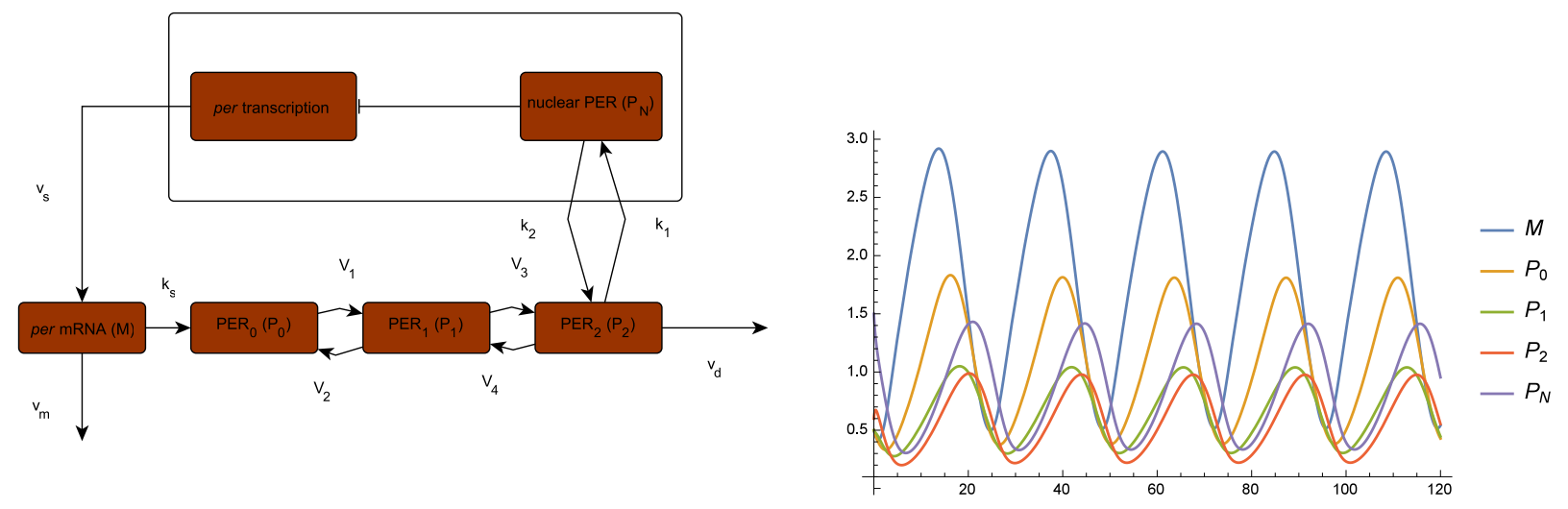

(a) Model for circadian PER oscillations, see [11]. Scheme (b) A plot of the model (1) to (5) with the parameters of the model for circadian oscillations of PER and per $v_{s}=0.76 \frac{\mu \mathrm{M}}{h}, v_{m}=0.65 \frac{\mu \mathrm{M}}{h}, K_{m}=0.5 \mu \mathrm{M}, k_{s}=0.38 \frac{1}{h}$, mRNA. The system of the equations is given by (1) to $v_{d}=0.95 \frac{\mu \mathrm{M}}{h}, k_{1}=1.9 \frac{1}{h}, k_{2}=1.3 \frac{1}{h}, K_{I}=1 \mu \mathrm{M}, K_{d}=$ (5). The per mRNA $(M)$ is transcribed in the nucleus $0.2 \mu \mathrm{M}, n=4, K_{1}=K_{2}=K_{3}=K_{4}=2 \mu \mathrm{M}, V_{1}=$ and is transported to the cytosol, where it accumulates $3.2 \frac{\mu \mathrm{M}}{h}, V_{2}=1.58 \frac{\mu \mathrm{M}}{h}, V_{3}=5 \frac{\mu \mathrm{M}}{h}, V_{4}=2.5 \frac{\mu \mathrm{M}}{h}$ with the at a maximum rate $v_{s}$; thereby it is degraded by an en- initial values $M(0)=0.5, P_{0}(0)=0.5, P_{1}(0)=0.5$, zyme of maximum rate $v_{m}$ and Michaelis constant $K_{m} \cdot P_{2}(0)=0.6, P_{N}(0)=1.5$.

The rate of translation of the PER protein, that is proportional to $M$, is characterized by a first-order rate constant $k_{s}$. The parameters $V_{i}$ and $K_{i}, i=1,2,3,4$, denote the maximum rate and Michaelis constant of the kinase(s) and phosphatase(s) involved in the reversible phosphorylation of $P_{0}$ into $P_{1}$ and $P_{1}$ into $P_{2}$ of the PER protein, respectively. The fully phosphorylated form $\left(P_{2}\right)$ is degraded by an enzyme of maximum rate $v_{d}$ and Michaelis constant $K_{d}$, and transferred into the nucleus at a rate characterized by the first-order rate constant $k_{1}$. Transport of the nuclear, biphosphorylated form of PER $\left(P_{N}\right)$ into the cytosol is characterized by the first-order rate constant $k_{2}$. The negative feedback exerted by nuclear PER on per transcription is described by an equation of the Hill type, in which $n$ denotes the degree of cooperativity and $K_{I}$ the threshold constant for inhibition.

Figure 1 
a coupling constant that weights the influence of the term $-u M$ on the time variation of $M$. For our experiment, we use $\alpha=0.05$.

Our first experiment is as follows. We assume light as our periodic external stimulus and we assume that the effect of this stimulus can be effectively modeled by an increased degradation of per mRNA. The light intensity oscillates with a period of 24 hours according to

$$
u(t):=\cos \left(2 \pi \frac{t}{24}+\varphi\right)+1
$$

where $\varphi$ is the time shift of external and internal time of the cell. We remark that there are more sophisticated ways to model the intensity of light, for example with a constant 0 over night and a peak about noon. However, this does not change the qualitative arguments in our analysis while making the study more complex. We start with $\varphi=0$. Then, the external stimulus has the same phase as the endogenous time of the cell, see Figure 2a, Figure $2 \mathrm{~b}$ and the curves almost look like the unperturbed ones from Figure 1b. That means that our framework is in accordance with the model proposed in [11]. Furthermore, we see that the period stays the same and is not changed by the mechanism that includes the external stimulus. Regarding the different forms of PER, we only show a detailed plot of the mRNA (Figure 2b) since the plots of all forms look the same. This is done throughout the following work. Later in the paper we discuss the consequences of the external period differing from the period of the endogenous clock.

If the external time is shifted compared to the endogenous time of the cell by for example $\varphi=\pi$, then we see in Figure 2c and Figure 2d that the endogenous time is shifted by approximately 12 hours after a transient of about 2 days. The external stimulus synchronizes the endogenous clock with the external time so that the phase of the cell's endogenous time is again in sync with the phase of the external time. We remark that the period of about 24 hours is unperturbed after the transient.

For illustration, we have the figures analogous to the results above for $\varphi=\frac{\pi}{2}$ and have a shift of about 6 hours after a transient, see Figure 2c and Figure $2 \mathrm{f}$.

\section{Specific organism models}

In the fly we have, mediated by a signal pathway, a degradation of the PER protein caused by the external stimulus effectively modeled with

$$
\frac{d}{d t} P_{0}=k_{s} M-V_{1} \frac{P_{0}}{K_{1}+P_{0}}+V_{2} \frac{P_{1}}{K_{2}+P_{1}}-\alpha u P_{0}
$$

where $\alpha>0$. We take our minimal model (1) to (5) where (2) is exchanged by (8) for flies. We show that, analogously to Supplementary Figure 4, the unperturbed rhythm is shifted by about 12 hours if one applies (16) with $\varphi=\pi$, see Figure 3.

In mammals we have instead that light induces per transcription. Therefore, we introduce a new model where we replace (1) by

$$
\frac{d}{d t} M=v_{s} \frac{K_{1}^{n}}{K_{1}^{n}+P_{N}^{n}}-v_{m} \frac{M}{K_{m}+M}+\gamma u \exp (-\varrho M)
$$

while we still use $(2)$ to $(5)$ with $\gamma, \varrho>0$. The term $\exp (-\varrho M)$ models the fact that if there is a lot of per mRNA then a light stimulus is supposed to be not that effective as it is when there is almost no per mRNA. In this work, we use $\varrho=1$. 


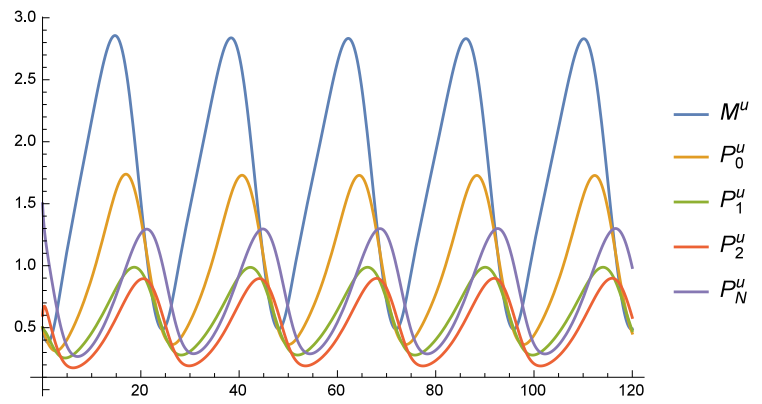

(a)

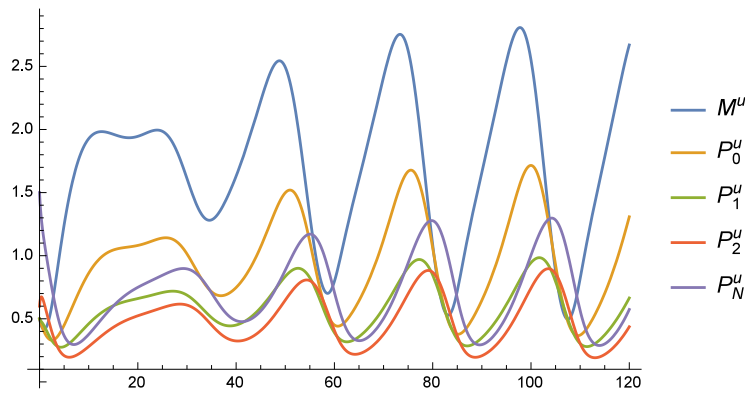

(c)

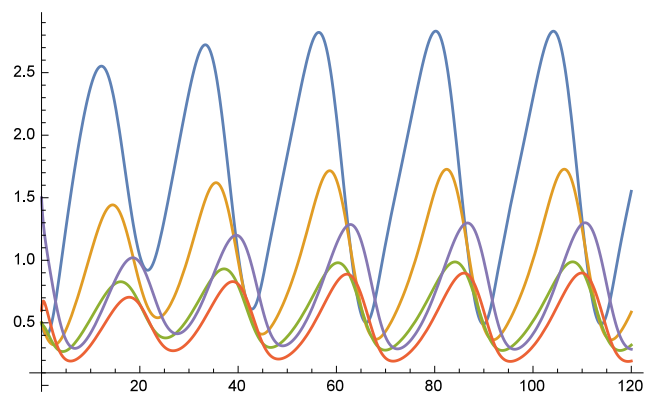

(e)

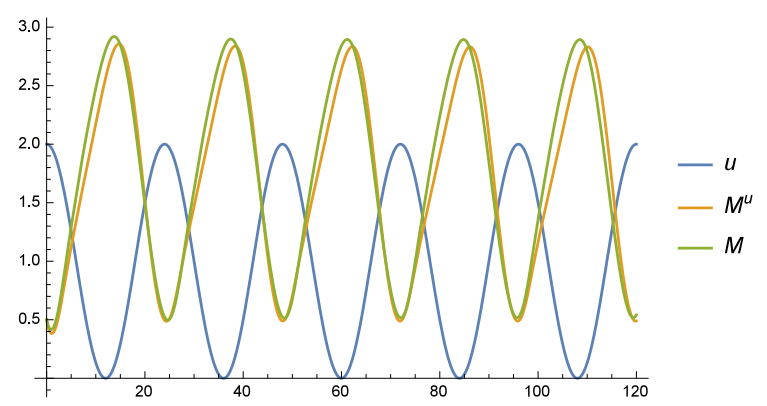

(b)

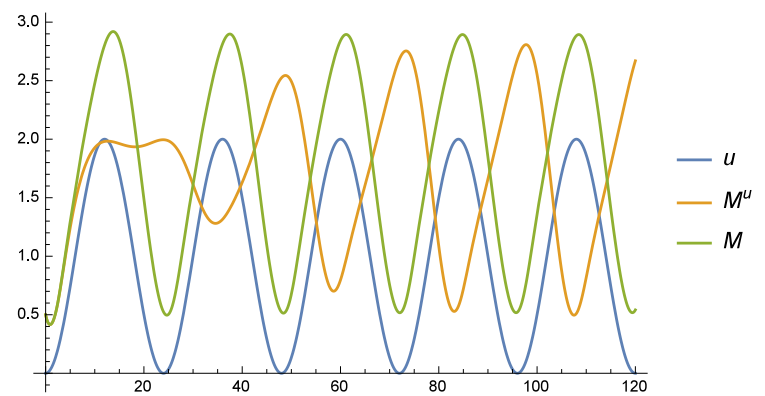

(d)

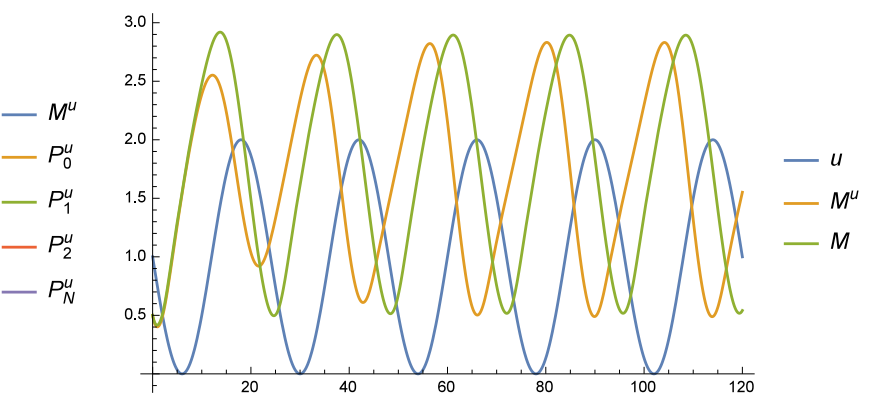

(f)

Figure 2: The model of Goldbeter including our extension does not differ from the original model of Goldbeter if the molecular clock is in the phase of the external Zeitgeber. (a) Time curves where the superscript $u$ indicates that these time curves stem from the extended model consisting of (6) and (2) to (5) with (7) for $\varphi=0$ and $\alpha=0.05$. (b) Time curve of the external stimulus $u$ defined in (7), of $M$ calculated from the model 1 to (5) and of $M^{u}$ calculated from (6) and (2) to (5) with (7) for $\varphi=0$ and $\alpha=0.05$. For the Figure (c) and (d) the external stimulus induces a shift of the molecular clock's phase of 12 hours where the experiment is as in (a) and (b) but with $\varphi=\pi$. The external stimulus induces a shift of the molecular clock's phase of about 6 hours in (e) and (f) where the experiment is as in (a) and (b) with $\varphi=\frac{\pi}{2}$. 


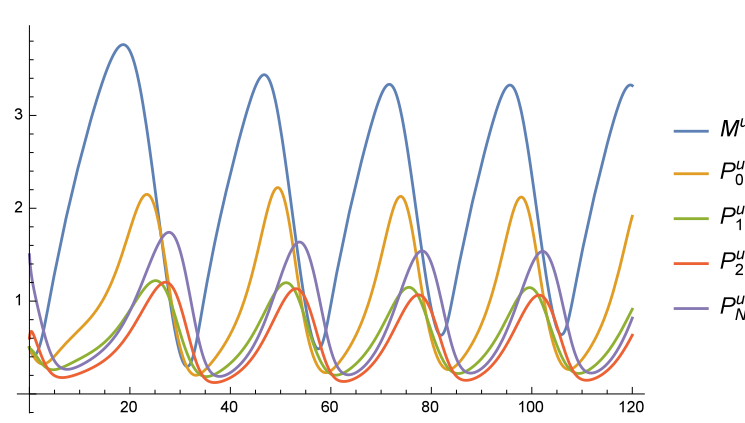

(a)

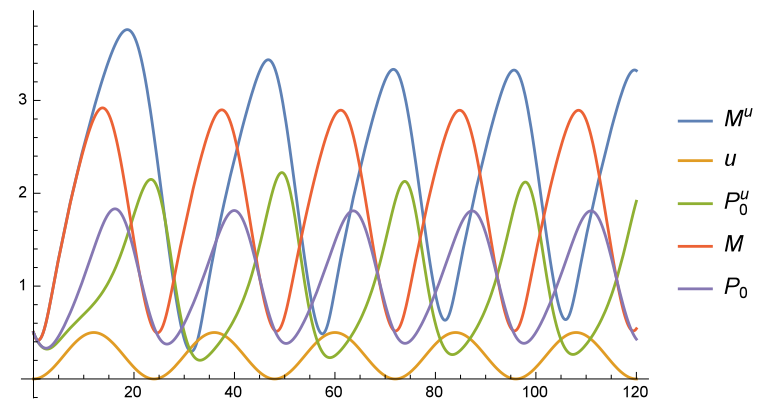

(b)

Figure 3: A further digestion of PER protein shifts the phase of the molecular clock. (a) Time curves where the superscript $u$ indicates that these time curves stem from the extended model consisting of (1) to (5) where (2) is replaced by (8) for (16) and $\alpha=1$. (b) Time curve of the external stimulus $u$ defined in (16), of $M$ and $P_{0}$ calculated from the model 1 to (5) and of $M^{u}$ and $P_{0}^{u}$ calculated from (1) to (5) where (2) is replaced by (8) for (16) and $\alpha=1$.

We remark that we assume that the production of mRNA $(M)$ is limited by the capacity of the transcription process. That means once this limit is reached more strength of external stimuli has no effect. We assume that $\exp (-\varrho M)$ models the fact that if there is much $M$, then there will be a lot of active transcription machinery. If we would like to avoid the exponential term and replace it with a bilinear mechanism with the same qualitative effect, we have to add a further equation modeling the transcription capacities. For example we have the species $T$ which is the unstimulated transcription machinery and $T^{*}$ which is stimulated one by the external stimulus. We have $\frac{d}{d t} T=-\varrho u T+\xi T^{*}$ and $\frac{d}{d t} T^{*}=\varrho u T-\xi T^{*}$ with $T(0)=T_{0}>0, \xi>0$ and $T^{*}(0)=0$ for example. The external stimulus $u$ transforms the unstimulated species into the stimulated one. The term $\xi T^{*}$ models the decay of $T^{*}$ or a self inhibition. By the fixed initial value $T_{0}$, the capacity of transcription machinery that can be stimulated is limited and we can replace $u \exp (-\varrho M)$ by $T^{*}$ in (9). Of course we can further extend this model by including a negative feedback loop into the equations for $T$ and $T^{*}$ which models the fact that mRNA limits its own transcription. This could be done be adding $M T^{*}$ into the equation for $T$ and subtract it in the equation for $T^{*}$ where each $M T^{*}$ is multiplied with the same constant. Depending on the quality of the data, one may also model the reaction mechanisms more detailed if there are several molecules binding to certain proteins of the transcription machinery or if there are cascades of reactions where different molecules bind to the same protein one after another. The smaller the error bars of the data points are, the more sophisticated models, i.e. with more parameters, can be tested and distinguished with respect to if they have to be rejected based on the data or not. To implement more sophisticated models we can use modified equations describing the dynamics according to the law of mass action for each species defined by the molecules that have already bound to the corresponding protein. Since the focus of this work is on the presentation of working systematically with external stimuli where all the previous discussed formulas fit into, we would like to proceed with the most easy model presented in (9) for reasons of clarity.

\section{Constant light}

How do we model a constant stimulus such as light? We choose a constant external stimulus, i.e.

$$
u_{c}(t)=c,
$$


that causes a degradation of $P_{0}$ (fly model) or a further transcription of $M$ (mouse model) where $c \geq 0$ is the intensity of the external stimulus. Again, we determine the periods by the peak of the oscillations of the per mRNA determined with the FindArgMax function of Mathematica in the range $t \in[80,120]$ hours.

We start with the fly model with $\alpha=1$ and obtain first a shortening of the period and from a turning point an elongation of the period the stronger the stimulus becomes until we have no oscillation any more which we call arrhythmic behavior. See Figure $4 \mathrm{a}$ and Figure $4 \mathrm{~b}$ for a strong intensity where $c=1$ causes an arrhythmic behavior, which means that the oscillations fade for a strong intensity of the external stimulus until there is no rhythm, and see Figure 4c and Figure $4 \mathrm{~d}$ for a weak intensity of the external stimulus where $c=0.5$ and the amplitude of the oscillations is smaller compared with the unperturbed oscillations.

Now, we investigate the mammalian model for $\gamma=2$. This causes that the model also shows arrhythmic behavior for $c=1$. We have that the period becomes shorter until $c=0.4$ and then the period becomes longer until the stimulus is so strong that no oscillation is detectable any more. The figures in this experiment look similar to the ones from the last experiment, but with different turning points, see Figure 4e.

We conclude from the two experiments that weak illumination (a small constant stimulus $c$ ) causes a shortening of the period, whereas high illumination (a larger $c$ ) causes a lengthening of the period. Whether and when period shortening or lengthening is observed depends on the model (fly or mammalian) and the corresponding parameters. In the fly model, the period shortening occurs only for a small range of $c$ (very low illumination) while the lengthening can be seen at a wide range of "c" (low to high illumination). This is exactly what was experimentally found in flies [18]. In the mammalian model, the turning point between period shortening and lengthening happens much later, so that shortening and lengthening cover almost equal ranges of $c$ (illumination). Consequently, constant light can shorten or lengthen the period of a mammal depending on the chosen illuminance. Natural light can vary between 0 and 100000 lux. In most experiments, only a small range of illuminance was tested and consequently, either period shortening or lengthening was observed [17]. Furthermore, the responses are different in different species and most likely depend on the light-sensitivity of their eyes. Nocturnal species that usually have very light-sensitive eyes are expected to show mainly period lengthening whereas diurnal species with less sensitive eyes should predominantly show period shortening. Indeed, Jürgen Aschoff observed exactly this phenomenon and it became known as "Aschoffs rule". Our model predicts these results. The specific mathematical argument is that models for nocturnal species have a larger $\alpha$ or $\gamma$ compared with the values used for the data plotted in Figure 4e. Looking closer at (8) or (9), we see that due to the product of $\alpha u$ or $\gamma u$, respectively, a smaller $u$ is needed in order to obtain the same coefficient in front of the term $P_{0}$ or $\exp (-\varrho M)$, respectively. This means that a weaker illuminance is needed to be right next to the turning point. The argumentation is analogous for diurnal species where $\alpha$ or $\gamma$ are smaller compared with the values used for the data plotted in Figure 4e.

Furthermore, considering [18, Table III] and our model, we have that different organisms or mutants have different ranges of intensity where a constant stimulus causes shortening or lengthening of the period of the endogenous clock. Thus if a period increases or decreases if the organism or mutant is exposed first to constant light and then constant darkness or the other way round, depends on whether the intensity of the constant light is in the range where the corresponding graph is below the free running period or above. We stress that especially with mutants the corresponding turning point might be at a so high intensity that a external stimulus cannot be applied without causing any damage to the considered organism in order to see both behaviors of the period, that means shortening and lengthening. 
We could easily adapt our model to different orders of magnitude for the illuminance by replacing $u$ by $\ln (1+u)$, for instance, adapting the corresponding parameter $\alpha$ or $\gamma$.

\section{Modeling central and peripheral oscillators}

In this section the presented framework of external stimuli is applied to model the signaling from the SCN to the peripheral tissues to synchronize the peripheral clocks [22]. In the next experiment, we show that several oscillators can be coupled by our framework that includes external stimuli as follows. A first oscillator responds to external stimuli, for example to light as the SCN. A second oscillator receives a signal from the first oscillator that correlates to an agent involved in the first oscillator and thus this signal acts as the "external stimulus" for the second oscillator. While for the SCN (first oscillator) the external stimulus is light, the external stimulus for the peripheral clock (second oscillator) can be hormones, neuronal activity or body temperature. If one of these signals correlates with the occurrence of per mRNA or of the (phosphorylated) PER protein, we can couple these two oscillators for example as follows.

We take the basic equations (1) to (5), where the first oscillator is sensitive to an external stimulus, that means (2) is replaced by (8). The second oscillator with its corresponding agents $\tilde{M}, \tilde{P}_{0}, \tilde{P}_{1}, \tilde{P}_{2}$ and $\tilde{P}_{N}$ is also modeled by analogous equations as (1) to (5) and is coupled to the first oscillator by also replacing $(2)$ by

$$
\frac{d}{d t} \tilde{P}_{0}=k_{s} \tilde{M}-V_{1} \frac{\tilde{P}_{0}}{K_{1}+\tilde{P}_{0}}+V_{2} \frac{\tilde{P}_{1}}{K_{2}+\tilde{P}_{1}}-\tilde{\alpha} \tilde{P}_{0} P_{0}
$$

with $\tilde{\alpha}>0$. That means there is an additional digestion of $\tilde{P}_{0}$ if the concentration of $P_{0}$ and $\tilde{P}_{0}$ is high at the same time. The concentration of $P_{0}$ is transmitted by for example some signaling pathway to the second oscillator. More specific, the further digestion of $\tilde{P}_{0}$ correlates to a high concentration of $P_{0}$ and $\tilde{P}_{0}$ at the same time. In Figure $5 \mathrm{a}$ and Figure $5 \mathrm{~b}$, we see that the second oscillator is synchronized to an antiparallel oscillation compared to the first one, that means that there is a phase shift of $\pi$ after the transient.

A parallel synchronization can be achieved by the following model

$$
\frac{d}{d t} \tilde{P}_{0}=k_{s} \tilde{M}-V_{1} \frac{\tilde{P}_{0}}{K_{1}+\tilde{P}_{0}}+V_{2} \frac{\tilde{P}_{1}}{K_{2}+\tilde{P}_{1}}-\tilde{\alpha} \tilde{P}_{0} \exp \left(-\beta P_{0}\right)
$$

where $\beta>0$. Here, in this model, a higher concentration of $P_{0}$ causes a lesser digestion of $\tilde{P}_{0}$, which is transmitted by some signaling pathway for example. More specific, a lesser digestion of $\tilde{P}_{0}$ correlates to a higher concentration of $P_{0}$. For the discussion of replacing the term $\exp \left(-\beta P_{0}\right)$ by alternative bilinear models, see the end of Section 4 below (9). Here the process can be analog where $P_{0}$ stimulates another protein which is transformed from an inactive to a stimulated species. Then the stimulated species, which is high if $P_{0}$ is low, can replace the term $\exp \left(-\beta P_{0}\right)$. For clarity we proceed with the (12) to demonstrate how to couple different small models to a big one with the idea of inputs modeled by external stimuli. In Figure 5c and Figure 5d, we see the synchronization of both oscillators with a shift of 0 after the transient.

If we take the model of two coupled oscillators mentioned above and replace (11) by

$$
\frac{d}{d t} \tilde{P}_{0}^{p}=k_{s} \tilde{M}-V_{1} \frac{\tilde{P}_{0}^{p}}{K_{1}+\tilde{P}_{0}^{p}}+V_{2} \frac{\tilde{P}_{1}^{p}}{K_{2}+\tilde{P}_{1}^{p}}-\alpha^{P} \tilde{P}_{0}^{p} P_{2}
$$




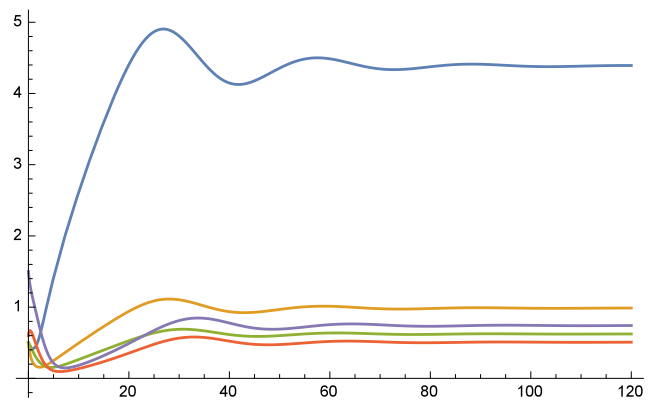

(a)

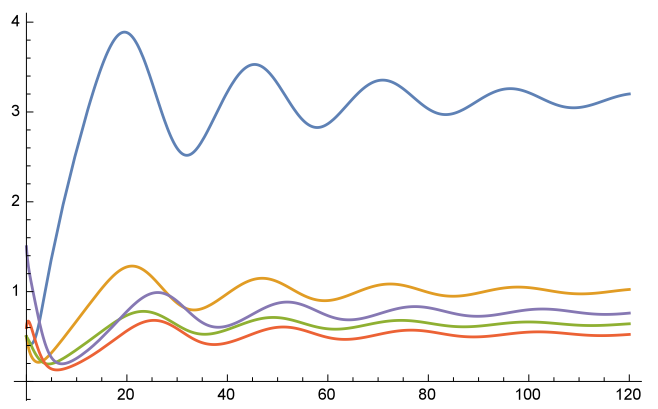

(c)

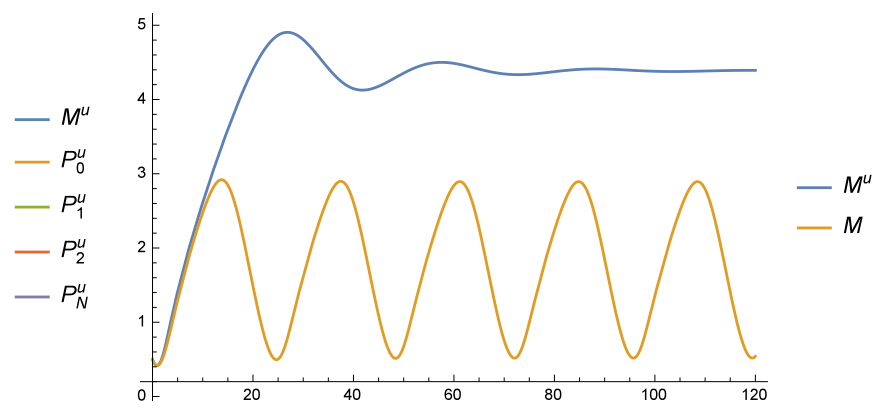

(b)

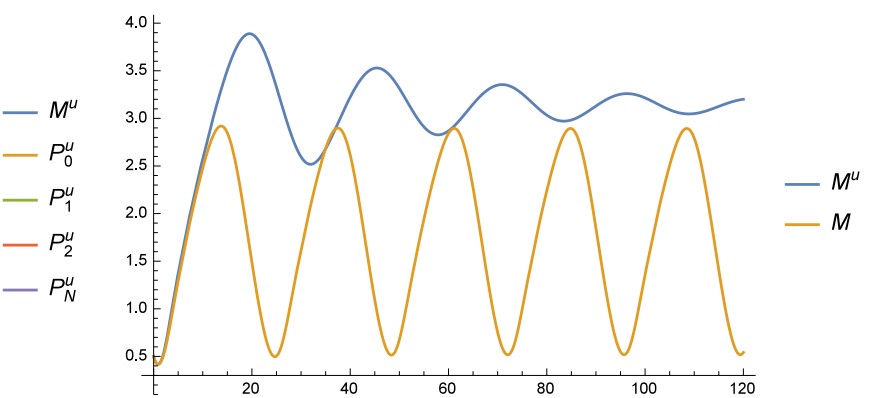

(d)

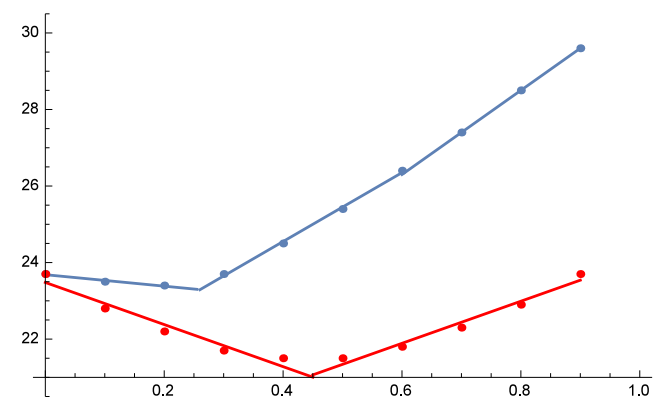

(e)

Figure 4: A strong permanent external stimulus causes arrhythmic behavior as the oscillations are dampened off. (a) Time curves where the superscript $u$ indicates that these time curves stem from the extended model consisting of (1) to (5) where (2) is replaced by (8) for (10) with $\alpha=1$ and $c=1$. (b) Time curve of $M$ calculated from the model 1 to (5) and of $M^{u}$ calculated from (1) to (5) where (2) is replaced by (8) for (10) with $\alpha=1$ and $c=1$. In (c) and (d) a weak permanent external stimulus causes dampening of the amplitude of the oscillations, however they are still measurable. The experiment is as in (a) and (b) with $c=0.5$. In (e) we have on the abscissa the illuminance $c$ and on the ordinate we have the period length in hours. The periods of the endogenous clocks of the fly and the mammalian model under a constant external stimulus. The blue graph is from the fly model and the red one is from the mammalian model. The data points for the fly model are $(0,23.7),(0.1,23.5),(0.2,23.4),(0.3,23.7)$, $(0.4,24.5),(0.5,25.4),(0.6,26.4),(0.7,27.4),(0.8,28.5),(0.9,29.6)$. The data points for the mammalian model are $(0,23.7),(0.1,22.8),(0.2,22.2),(0.3,21.7),(0.4,21.5),(0.5,21.5),(0.6,21.8)$, $(0.7,22.3),(0.8,22.9),(0.9,23.7)$. 
where the additional decay of $\tilde{P}_{0}^{p}$ depends on the product of $\tilde{P}_{0}^{p}$ and $P_{2}$ instead of $\tilde{P}_{0}$ and $P_{0}$ as in (11), then we can see in Figure 5e that the shift of $P_{2}$ compared to $P_{0}$ provides a system of two coupled oscillators where the shift of phase can have more values than just inphase courses, i.e. a phase shift of 0 , see Figure $5 \mathrm{c}$ or antiphase courses, i.e. a phase shift of $\pi$, see Figure 5 a. By this mechanism of coupling different oscillators, nature might create peripheral endogenous clocks which are out of phase with the clock of the SCN for a varying amount of hours. Thus they may adopt any phases that are advantageous for the physiological function of the peripheral oscillator.

\section{$7 \quad$ Food entrained peripheral clocks}

In our next experiment, we model a similar situation as described for the liver and SCN clocks of mammals. As already described, the SCN coordinates the phases of peripheral clocks, such as that in the liver [22]. But the SCN is not the only Zeitgeber for the liver clock, food intake is another important Zeitgeber. Under normal conditions both Zeitgebers are in phase with each other. However, when food is only available outside the animal's normal activity time, feeding can phase shift the liver clock, but not the SCN clock, so that both clocks can take an unusual phase relationship [10]. We choose the model that is used for the experiment depicted in Figure 5c. Furthermore, we equip (12) with an additional term that models the stimulus that is caused by the food intake as follows

$$
\frac{d}{d t} \tilde{P}_{0}=k_{s} \tilde{M}-V_{1} \frac{\tilde{P}_{0}}{K_{1}+\tilde{P}_{0}}+V_{2} \frac{\tilde{P}_{1}}{K_{2}+\tilde{P}_{1}}-\tilde{\alpha} \tilde{P}_{0} \exp \left(-\beta P_{0}\right)-\delta \tilde{u} \tilde{P}_{0}
$$

with $\delta>0$ where the external stimulus is given by

$$
\tilde{u}(t):= \begin{cases}\frac{1}{4}\left(\cos \left(2 \pi \frac{t}{24}+\tilde{\varphi}\right)+1\right) & \text { if } t \leq \tilde{t} \\ u(t) & \text { else }\end{cases}
$$

with $u(t)$ defined by

$$
u(t):=\frac{1}{4}\left(\cos \left(2 \pi \frac{t}{24}+\varphi\right)+1\right)
$$

and $\tilde{t}>0$. Equation (14) induces a further digestion of $\tilde{P}_{0}$ by the term $-\delta \tilde{u} \tilde{P}_{0}$. More specific, the model considers the case that food intake correlates with the digestion of $\tilde{P}_{0}$. We choose the external stimulus $u$ such that it is inphase with the SCN, that means $\varphi=0$ in (16) which is associated with day light. Now we choose $\tilde{\varphi}=\pi$ as food intake is supposed to happen at night instead of the day which corresponds to $\tilde{\varphi}=0$. That means that until $\tilde{t}$ food intake happens at night and after $\tilde{t}$ food intake is at day time.

In Figure 6 we only depict the time curves for per mRNA to keep the figure clear for $\tilde{t}=160$. We see that the phase of the peripheral clock $(\tilde{M})$ is shifted by about 6 hours after the transient compared to the phase of the central clock $\left(M^{u}\right)$ when food intake happens at night. When the food intake takes place again at day time as in Figure 6 after $t=160$, i.e. there is no shift between $u$ and $\tilde{u}$, then the peripheral clock is synchronized to the central clock again after a transient of about 1 day.

The effect on the peripheral oscillator can be interpreted as follows. All the external stimuli or signal inputs, respectively, are integrated by the peripheral clock. The optimal time point for the 


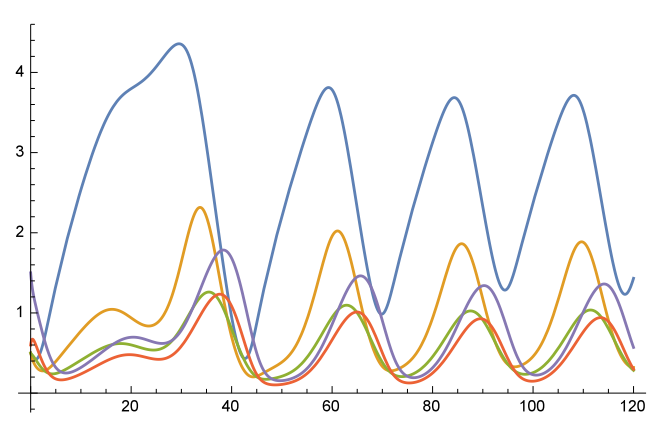

(a)

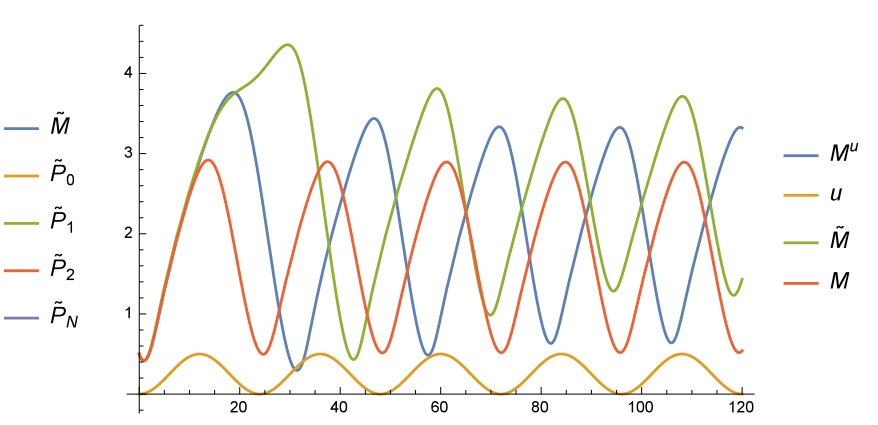

(b)

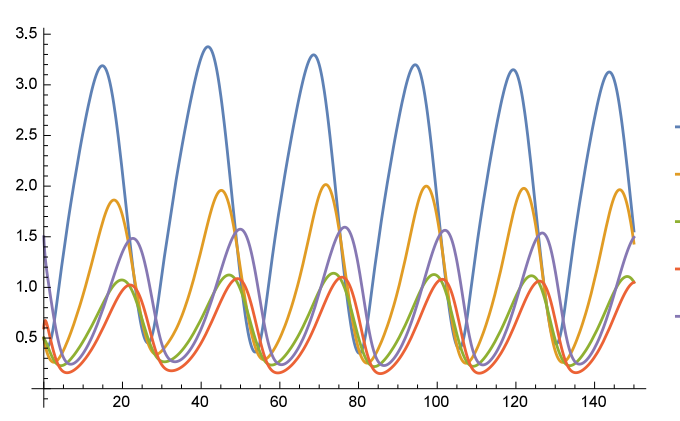

(c)

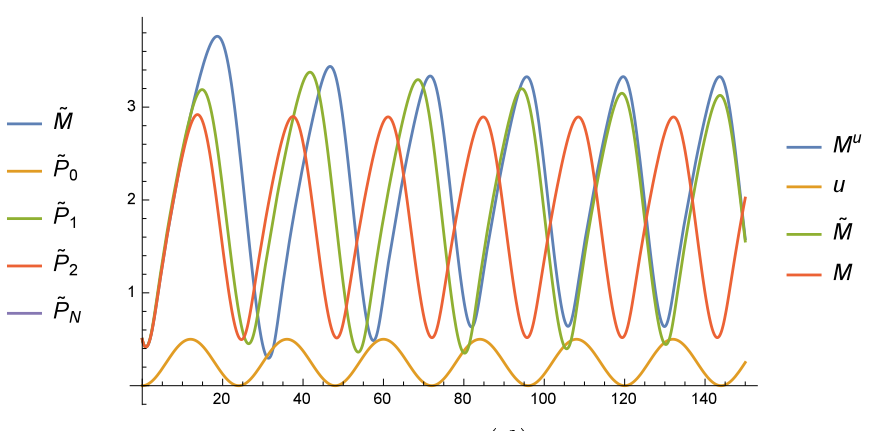

(d)

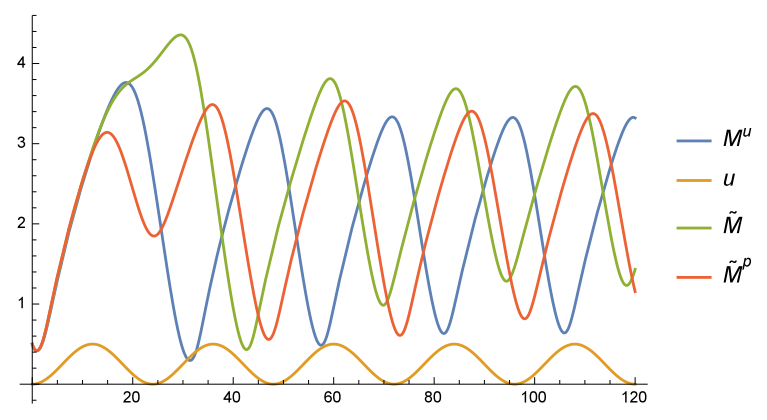

(e)

Figure 5: A peripheral oscillator coupled in antiphase to the molecular clock. (a) Time curves of the second oscillator where the agents $\tilde{M}, \tilde{P}_{0}, \tilde{P}_{1}, \tilde{P}_{2}$ and $\tilde{P}_{N}$ fulfill analog equations corresponding to (1) to (5) where (2) is replaced by (11) and $\tilde{\alpha}=0.5$. (b) Time curve of the external stimulus $u$ defined in (16), of $M$ calculated from the model (1) to (5), of $\tilde{M}$ calculated by an analogous model like (1) to (5) where (2) is replaced by (11) with $\tilde{\alpha}=0.5$ and of $M^{u}$ calculated from (1) to (5) where (2) is replaced by (8) with $\alpha=1$ for (16). In (c) and (d) a peripheral oscillator coupled in phase to the molecular clock. (c) Time curves of the second oscillator where the agents $\tilde{M}_{0} \tilde{P}_{0}, \tilde{P}_{1}$, $\tilde{P}_{2}$ and $\tilde{P}_{N}$ fulfill analog equations corresponding to (1) to (5) where (2) is replaced by (12) with $\tilde{\alpha}=0.5, \beta=2$. (d) Time curve of the external stimulus $u$ defined in (16), of $M$ calculated from the model (1) to (5), of $\tilde{M}$ calculated by an analogous model like (1) to (5) where (2) is replaced by (12) with $\tilde{\alpha}=0.5, \beta=2$ and of $M^{u}$ calculated from (1) to (5) where (2) is replaced by (8) with $\alpha=1$ for (16). In (e) the time curve of the external stimulus $u$ defined in (16) where $M^{u}$ and $\tilde{M}$ are calculated as in (a) and (b). The quantity $\tilde{M}^{p}$ is calculated by an analogous model as $\tilde{M}$ where (11) is replaced by (13) with $\alpha^{P}=0.5$. After the transient, we have a shift of about 12.7 hours between $M^{u}$ and $\tilde{M}$ and of about 15.8 hours between $M^{u}$ and $\tilde{M}^{p}$. 


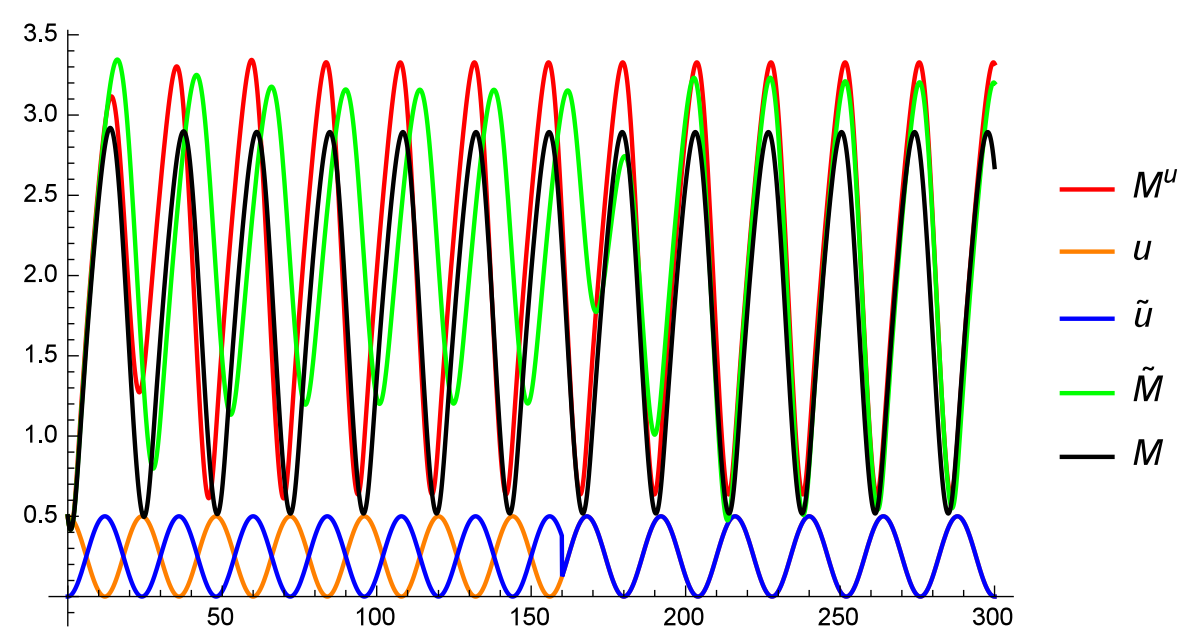

Figure 6: Time curve of the external stimulus $u$ defined in (16) and $\tilde{u}$ defined in (15) with $\tilde{t}=160$ and $\tilde{\varphi}=\pi$, of $M$ calculated from the model 1 to (5), of $\tilde{M}$ calculated by an analogous model like (1) to (5) where (2) is replaced by (14) with $\tilde{\alpha}=0.3, \beta=2, \delta=0.4$ and of $M^{u}$ calculated from (1) to (5) where (2) is replaced by (8) with $\alpha=1$ for (16) with $\varphi=0$.

liver cell to be active and to fulfill the intended tasks in the body is triggered by the input and importance (strength) of the external stimuli or signal inputs received from the SCN and feeding. Reacting on different external stimuli at the same time according to a mechanism mentioned above in (14) can also be seen as making a compromise to meet all the circumstances at the same time that correspond to the simultaneously emerging external stimuli.

Notice that the closer $\tilde{\varphi}$ is to $\varphi$ the smaller the shift of the phases of the two oscillators is and the stronger the stimulus $\tilde{u}$ is the closer the shift of the phases of the two oscillators comes to the phase shift between $u$ and $\tilde{u}$.

\section{Discussion}

We start our discussion by briefly outlining the novelty of the present work before we give more details and relate our work to existing ones. Our simulation starts with a minimal oscillator model and it is extended by an external stimuli framework that has not been used before (see $[11,33,31,34,15])$. This framework allows to analyze the behavior of different external stimuli in order to demonstrate the generality and the practicability of the framework that allows easily to include external stimuli exemplified for circadian clocks and oscillator coupling. Further the novelty is especially in modeling several effects reported in experimental works just by our unified and comprehensive model. The model is mathematical consistent and uses an enzyme kinetic formalism based on the law of mass action. For this reason each equation can be investigated on its own by taking the external stimuli as an input function with given values. Then the behavior of the corresponding quantity modeled with this equation can be studied upon the external stimuli, ranging from e.g. numerical stability to effects of the external stimuli to the output values which are the values of the corresponding quantity. We do not intended to generate an animal-specific model. To achieve this, the works listed in Table 2 have to be specifically considered and the formalisms listed there added to our model, for instance the van der Pol Oscillator which is a very basal model for understanding oscillators with non-linear dynamics. 
Our model provides entrainment to the phase of a periodic external stimulus and the restart of the circadian rhythm upon inhibition of transcription or translation. Different effects of external stimuli to molecular agents are investigated to set up a fly and a mammalian model that are included in our framework. The fly model has a wide range to adapt to different periods of the external stimulus while the mammalian model provides a rather short range. Behavior under constant light is investigated for both models as well as phase-response curves are calculated. It is also shown how to couple different oscillators with the presented framework to model peripheral oscillators that are coupled to a central clock and integrate and receive additional signals to process all the information incoming at once. This procedure of coupling can be extended to any model and is a helpful tool, easy to implement, to study crosstalks and network effects of coupled biological systems. In this way the modeling works module wise since sub systems can be combined together to a total system by using outputs of the sub systems as inputs for others via the proposed external stimuli framework.

The circadian clock can be modeled by a system of ordinary differential equations that stem from the theory of enzyme kinetics which shows a stable rhythmic oscillation with a period of about 24 hours, see for example [11]. In our work, we aim to extend this model with additional terms modeling the effect of external stimuli like light, which causes activation or inhibition of transcription or translations as well as the degradation of proteins. We demonstrate that this model is in accordance with experimental results like entrainment of different periods in a range around 24 hours, phase-response curves or changing periods under constant light. Furthermore, our model aims at the molecular effects that determine the behavior of the oscillator upon external stimuli. By external stimuli we mean any agent that effects the corresponding molecular network like light, hormones or any other chemical agent. The framework allows to investigate also various other effects reported in literature, see Table 1 for instance.

There is previous work where theoretical models are presented that describe the effects of external stimuli to circadian oscillators. In [34, 31, 33, 35] they model the external stimuli by changing model and reaction parameters. However, we model the change of mRNA and protein levels caused by external stimuli by including additional terms into the unperturbed oscillator model. The model of [11] exhibits a limit cycle with a period of 24 hours only for a certain range of parameters. If the parameters are changed too much, the concentration of the molecular agents adjusts to a constant value as reported in [33]. Our framework does not show such a behavior because we model the external stimuli by extra terms instead of changing model parameters of the basic system that is responsible for a stable limit cycle. In our approach, we focus on effective models that decrease or increase the concentration of per mRNA or PER protein according to the effect of a stimulus and thus covers the effect of molecular mechanisms. By the word "effective" we mean that our model is a simple, clear and elegant one that includes only the essential information. Moreover, we have a straight forward way how to include experimental procedures like blocking translation as it is done in [15], for example.

The following works [34, 31, 33] calculate phase-response curves where in [31] also the limited entrainment to different external periods is discussed. These results are in accordance to our results, see the Supplementary material, where we focus also on the explanation of the effect that internal periods change according to permanent external stimuli. For this purpose, we use the model of [11] with our framework of external stimuli to show that different molecular effects of the permanent stimulus result in a different behavior with respect to constant light as reported in [17] for example (see "Constant light" under Results). These different effects are the degradation of PER protein and the increase of the per transcription. Additionally, we show (in the Supplementary material) that the endogenous clock modeled by [11] extended by our external stimuli framework 
can be stopped and thus can be restarted if the corresponding stimulus declines as reported in [15]. Also by this experiment, we see that the model proposed by [11] in combination with our framework that includes external stimuli is very robust with respect to perturbations as also for strong perturbations the oscillations with a period of about 23.7 hours start again if the external stimulus declines. We also show that our model can entrain its phase to the one of an external Zeitgeber that is straightforward included in our presented framework.

It is also demonstrated in the results part how our effective model of external stimuli can be utilized to couple different oscillators, central and peripheral oscillators, see [22] for biological examples. For the coupling mechanisms, our proposed framework of external stimuli is very useful as it focuses on the effect of the external stimuli to model not only light but also chemical agents, especially neuropeptide hormones which also have a significant effect on entrainment of peripheral clocks caused by signaling from the SCN, see for example [36, 3]. In our framework, we consider the oscillators as a macroscopic object that produces stable oscillations by molecular mechanisms whereas in [26] the focus is on a multioscillatory approach of coupled oscillators that provides a stable output oscillation. We also assume that our system is robust against noise or in other words the noise is that small and that it does not prevent the system from producing stable oscillations. In $[33,37]$ the issue is addressed with stochastic models how stable oscillations can exhibit in spite of noise. We remark that our framework can also be applied to stochastic models. Furthermore, our framework of implementing external stimuli is not restricted to the special model presented in [11] but can be applied to any model consisting of differential equations, like [34, 31, 32, 26], to set up an effective model that considers the effect of external stimuli to certain agents of the network. Therefore, our framework is generic and has a high explanation power that extends the mentioned work in a new way.

Moreover, our framework also provides a principle mechanism how to explain the effects referred to singularity where one pulse of light can stop the oscillation and another can start the oscillation again, see [38] for further reading. Two concepts can be used for explaining this. The first possibility is to use a macroscopic model that describes a population of microscopic oscillators as a single macroscopic oscillator. To describe the singularity the corresponding equations have to contain a point of rest, sometimes called steady state, see for example [39] for a system of differential equations having several steady states. If the external stimulus brings the system sufficiently close to that steady state, then the system converges to that point of rest as shown in [13]. This means that at this special point the values of the agents' concentration are such that their variation with respect to time is (almost) zero and thus there are no oscillations anymore. If a second pulse of an external stimulus brings the system sufficiently away from the steady state, the oscillations start again. However, in our model based on Goldbeter's one we have not observed such a behavior of a steady state in addition to the limit circle. A second possibility to describe the effect of singularity is to consider several microscopic oscillators whose output then is added together to a macroscopic oscillation. The key is that these microscopic oscillators are coupled inhomogeneously to the external stimulus that means that each couple constant defers a little bit. This can be for example because the cells are exposed differently to light because they are covered differently by tissue. It can be shown with our model that already five oscillators that have the same phase at the beginning get out of phase upon a one hour pulse of external stimulus depending on its strength and at which phase time it is given. Then the total macroscopic oscillation is weaker as the microscopic oscillators have different phases caused by their inhomogeneous coupling to the external stimulus and thus the addition of all outputs provides a smaller amplitude since, for instance, one oscillator is at the lowest point of its oscillation and another is at its highest point. A second pulse brings all the microscopic oscillators to approximately the same phase again such 
that the macroscopic oscillation has the initial amplitude. This works because the microscopic oscillators are all influenced differently according to their phase at the time when the second pulse of the external stimulus is given such that after this pulse all the microscopic oscillators are (almost)

in the same phase again. Simulations with five coupled oscillators can be found in [40, Point of singularity, page $47 \mathrm{ff}$.$] .$

\section{Conclusion}

We present a framework to model how external stimuli influence the circadian clock and how they can synchronize the rhythm to the external time phase. We extend the original model of Goldbeter et al. [11] to a general framework of external stimuli. We analyze it with respect to its behavior upon different external stimuli in order to demonstrate the versatility, generality and application of our proposed external stimuli oscillator response model.

We show how to model and investigate different molecular effects of a permanent stimulus and how it results in a different behavior with respect to constant light, light-dark cycles and phase shifts by strong stimuli. Our effective model of external stimuli can be utilized to obtain phase response curves, couple central and peripherals oscillators and look at food entrained peripheral clocks which serves as a blueprint for module wise modeling by assemble a total biological system by sub systems using outputs of a sub system as an input for another sub system. Moreover, our framework divides a biological system into sub systems considering the process of modeling these sub systems as creating functions mapping input information to output information where the external stimuli are the interfaces. The interface consists of the input information from the environment which is given into the considered biological system and the output information of the system which evolves according to the dynamic of the biological system. The dynamic is captured in the corresponding mathematical model. In particular, our framework provides a principle method how to investigate and explain the effects on biological oscillators referred to singularity where one pulse of light can stop the oscillation and another one can start the oscillation again by the inhomogeneous coupling of many oscillators to an external stimulus. The model allows to investigate also effects on circadian clocks such as relative coordination which means that the period of the circadian clock adapts to the period of the external stimulation in a certain range where outside this range the period of the circadian clock is not effected by the period of the external stimulation.

In summary, our model allows to elegantly achieve a semi quantitative description of diverse experimental effects on the circadian clock or a general biological oscillator upon external stimuli like light. This is comprehensively described here with a simple but effective mathematical model that contains differential equations that exhibit a solution that oscillates with a period of about 24 hours and terms that increase or decrease the concentration of a corresponding molecular agent of the oscillator, depending on the intensity of the corresponding stimulus and the agent's concentration. All scripts and the framework are available with a tutorial at https://www.biozentrum.uniwuerzburg.de/bioinfo/computing/circadian/.

\section{Methods}

We used the Wolfram Mathematica software, mainly the NDSolve function, to solve all the systems of ordinary differential equations of which our models consist. 
The Mathematica files The Mathematica files from

https://www.biozentrum.uni-wuerzburg.de/bioinfo/computing/circadian/ can be used for the starting point of investigations. The files are set up to solve ordinary differential equations and plots the corresponding time curves. Once the user has replaced the current model by their model, the files can immediately be used. In addition the Mathematica files can be used as a blueprint to easily build a new environment in any software that can solve ordinary differential equations numerically.

\section{Acknowledgment}

We thank Wolfgang Engelmann, Anders Johnsson and Karl-Heinz Witte for their helpful comments and discussions during the creation of this work. TD acknowledges funding by the Land of Bavaria (Bavarian contribution to DFG project - 324392634 - TRR 221/INF).

\section{Author contributions}

TB did the modeling, the implementation of the models with Mathematica and was involved in writing. CF contributed the a detailed expertise of molecular circadian clocks and was involved in writing. TD lead the study and was involved in writing.

\section{Conflict of Interest}

There are no conflicts of interest.

\section{References}

[1] Moore-Ede MC, Sulzman FM, Fuller CA. The clocks that time us: physiology of the circadian timing system. Harvard Univ. Pr.; 1982.

[2] Hardin PE, Panda S. Circadian timekeeping and output mechanisms in animals. Current opinion in neurobiology. 2013;23(5):724-731.

[3] Buhr ED, Takahashi JS. Molecular components of the Mammalian circadian clock. In: Circadian clocks. Springer; 2013. p. 3-27.

[4] Ceriani MF, Darlington TK, Staknis D, Más P, Petti AA, Weitz CJ, et al. Light-dependent sequestration of TIMELESS by CRYPTOCHROME. Science. 1999;285(5427):553-556.

[5] Vaidya AT, Top D, Manahan CC, Tokuda JM, Zhang S, Pollack L, et al. Flavin reduction activates Drosophila CRYPTOCHROME. Proceedings of the National Academy of Sciences. 2013;110(51):20455-20460.

[6] Emery P, Stanewsky R, Helfrich-Förster C, Emery-Le M, Hall JC, Rosbash M. Drosophila CRY is a deep brain circadian photoreceptor. Neuron. 2000;26(2):493-504.

[7] Plautz JD, Kaneko M, Hall JC, Kay SA. Independent photoreceptive circadian clocks throughout Drosophila. Science. 1997;278(5343):1632-1635. 
[8] Golombek DA, Rosenstein RE. Physiology of circadian entrainment. Physiological reviews. 2010;90(3):1063-1102.

[9] Lowrey PL, Takahashi JS. Mammalian circadian biology: elucidating genome-wide levels of temporal organization. Annu Rev Genomics Hum Genet. 2004;5:407-441.

[10] Damiola F, Le Minh N, Preitner N, Kornmann B, Fleury-Olela F, Schibler U. Restricted feeding uncouples circadian oscillators in peripheral tissues from the central pacemaker in the Suprachiasmatic Nucleus. Genes \& development. 2000;14(23):2950-2961.

[11] Goldbeter A. A model for circadian oscillations in the Drosophila period protein (PER). Proc R Soc Lond B. 1995;261(1362):319-324.

[12] Breitenbach T, Lorenz K, Dandekar T. How to Steer and Control ERK and the ERK Signaling Cascade Exemplified by Looking at Cardiac Insufficiency. International journal of molecular sciences. 2019;20(9):2179.

[13] Breitenbach T, Liang C, Beyersdorf N, Dandekar T. Analyzing pharmacological intervention points: A method to calculate external stimuli to switch between steady states in regulatory networks. PLoS computational biology. 2019;15(7):e1007075.

[14] Husse J, Eichele G, Oster H. Synchronization of the mammalian circadian timing system: Light can control peripheral clocks independently of the SCN clock. Bioessays. 2015;37(10):1119-1128.

[15] Yamaguchi S, Isejima H, Matsuo T, Okura R, Yagita K, Kobayashi M, et al. Synchronization of cellular clocks in the Suprachiasmatic Nucleus. Science. 2003;302(5649):1408-1412.

[16] Aschoff J. Circadian timing. Annals of the New York Academy of Sciences. 1984;423(1):442468.

[17] Aschoff J. Freerunning and entrained circadian rhythms. In: Biological rhythms. Springer; 1981. p. 81-93.

[18] Konopka RJ, Pittendrigh C, Orr D. Reciprocal behaviour associated with altered homeostasis and photosensitivity of Drosophila clock mutants. Journal of neurogenetics. 1989;6(1):1-10.

[19] Saunders DS, Thomson EJ. 'Strong' phase response curve for the circadian rhythm of locomotor activity in a Cockroach (Nauphoeta cinerea). Nature. 1977;270(5634):241.

[20] Pendergast JS, Friday RC, Yamazaki S. Photic entrainment of period mutant mice is predicted from their phase response curves. Journal of Neuroscience. 2010;30(36):12179-12184.

[21] Saunders DS, Gillanders SW, Lewis RD. Light-pulse phase response curves for the locomotor activity rhythm in period mutants of Drosophila melanogaster. Journal of Insect Physiology. 1994;40(11):957-968.

[22] Hastings MH, Reddy AB, Maywood ES. A clockwork web: Circadian timing in brain and periphery, in health and disease. Nature Reviews Neuroscience. 2003;4(8):649. 
[23] Leloup JC, Goldbeter A. A model for circadian rhythms in Drosophila incorporating the formation of a complex between the PER and TIM proteins. Journal of biological rhythms. 1998;13(1):70-87.

[24] Strogatz SH. Human sleep and circadian rhythms: a simple model based on two coupled oscillators. Journal of mathematical biology. 1987;25(3):327-347.

[25] Kronauer RE, Czeisler CA, Pilato SF, Moore-Ede MC, Weitzman ED. Mathematical model of the human circadian system with two interacting oscillators. American Journal of PhysiologyRegulatory, Integrative and Comparative Physiology. 1982;242(1):R3-R17.

[26] Diez-Noguera A. A functional model of the circadian system based on the degree of intercommunication in a complex system. American Journal of Physiology-Regulatory, Integrative and Comparative Physiology. 1994;267(4):R1118-R1135.

[27] Bordyugov G, Westermark PO, Korenčič A, Bernard S, Herzel H. Mathematical modeling in chronobiology. In: Circadian clocks. Springer; 2013. p. 335-357.

[28] Forger DB, Dean DA, Gurdziel K, Leloup JC, Lee C, Von Gall C, et al. Development and validation of computational models for mammalian circadian oscillators. OMICS A Journal of Integrative Biology. 2003;7(4):387-400.

[29] Leloup JC, Goldbeter A. Toward a detailed computational model for the mammalian circadian clock. Proceedings of the National Academy of Sciences. 2003;100(12):7051-7056.

[30] Forger DB, Peskin CS. A detailed predictive model of the mammalian circadian clock. Proceedings of the National Academy of Sciences. 2003;100(25):14806-14811.

[31] olde Scheper T, Klinkenberg D, Pennartz C, Van Pelt J. A mathematical model for the intracellular circadian rhythm generator. Journal of Neuroscience. 1999;19(1):40-47.

[32] Gonze D. Modeling circadian clocks: From equations to oscillations. Central European Journal of Biology. 2011;6(5):699.

[33] Leloup JC, Gonze D, Goldbeter A. Computational models for circadian rhythms: Deterministic versus stochastic approaches. In: Computational systems biology. Elsevier; 2006. p. 249-291.

[34] Petri B, Stengl M. Phase response curves of a molecular model oscillator: implications for mutual coupling of paired oscillators. Journal of Biological Rhythms. 2001;16(2):125-141.

[35] Kurosawa G, Goldbeter A. Amplitude of circadian oscillations entrained by 24-h light-dark cycles. Journal of theoretical biology. 2006;242(2):478-488.

[36] Saper CB, Scammell TE, Lu J. Hypothalamic regulation of sleep and circadian rhythms. Nature. 2005;437(7063):1257.

[37] Gonze D. Modeling circadian clocks: roles, advantages, and limitations. Open Life Sciences. 2011;6(5):712-729.

[38] Engelmann W, Witte KH. How to stop a biological clock: Point of singularity. Universitätsbibliothek Tübingen; 2016. 
[39] Di Cara A, Garg A, De Micheli G, Xenarios I, Mendoza L. Dynamic simulation of regulatory networks using SQUAD. BMC bioinformatics. 2007;8(1):462.

[40] Breitenbach T. A mathematical optimal control based approach to pharmacological modulation with regulatory networks and external stimuli [doctoralthesis]. Universität Würzburg; 2019. Available from: https://opus.bibliothek.uni-wuerzburg.de/frontdoor/ index/index/searchtype/authorsearch/author/Tim+Breitenbach/start/0/rows/1/ institutefq/Theodor-Boveri-Institut+f\%C3\%BCr+Biowissenschaften/docId/17436.

[41] Pittendrigh CS, Daan S. A Functional Analysis of Circadian Pacemakers in Nocturnal Rodents. IV. Entrainment: Pacemaker as Clock. Journal of Comparative Physiology A. 1976;106(3):291-331. Relation: https://www.rug.nl/fmns-research/cbn/index Rights: University of Groningen, Centre for Behaviour and Neurosciences. 
Figures
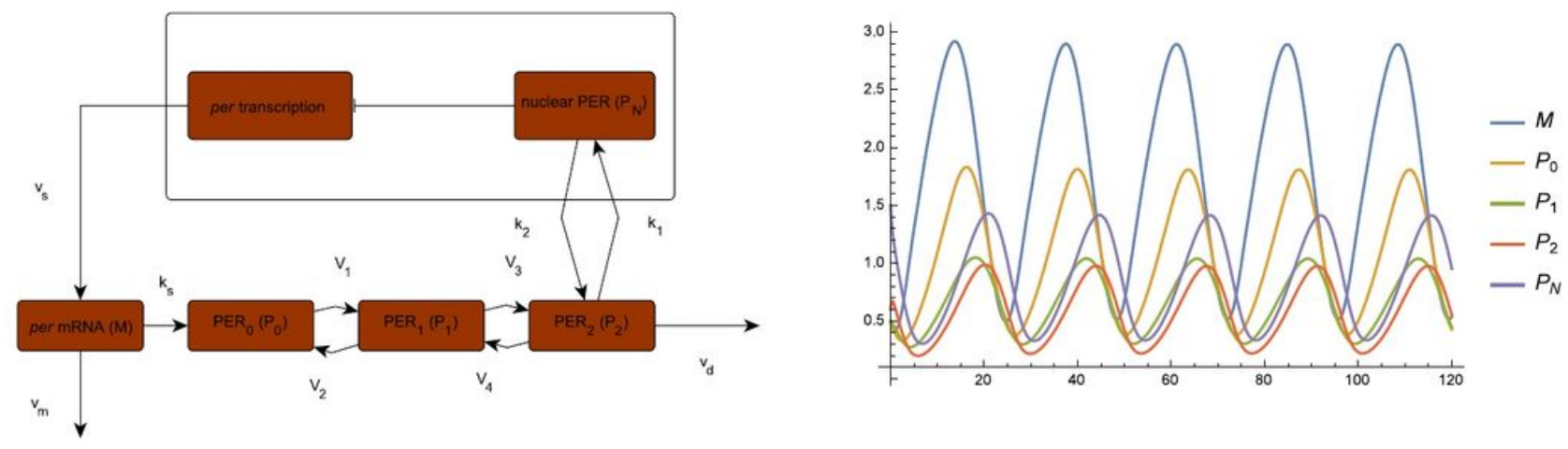

Figure 1

please see the manuscript file for the full caption 


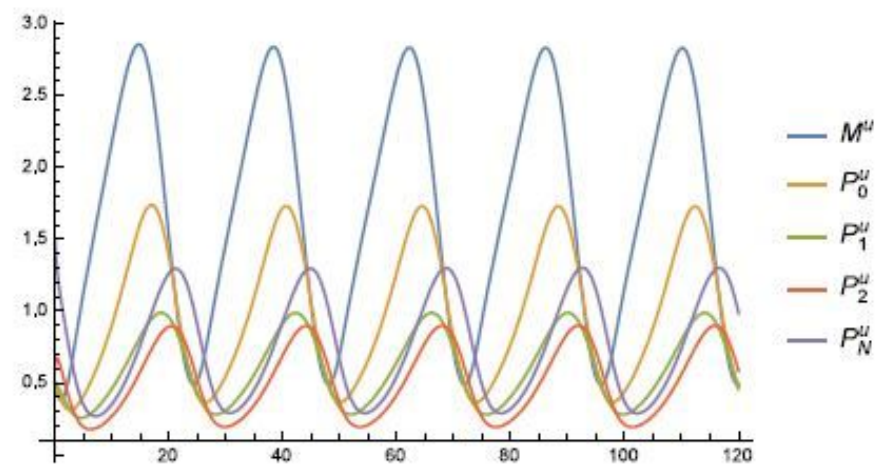

(a)

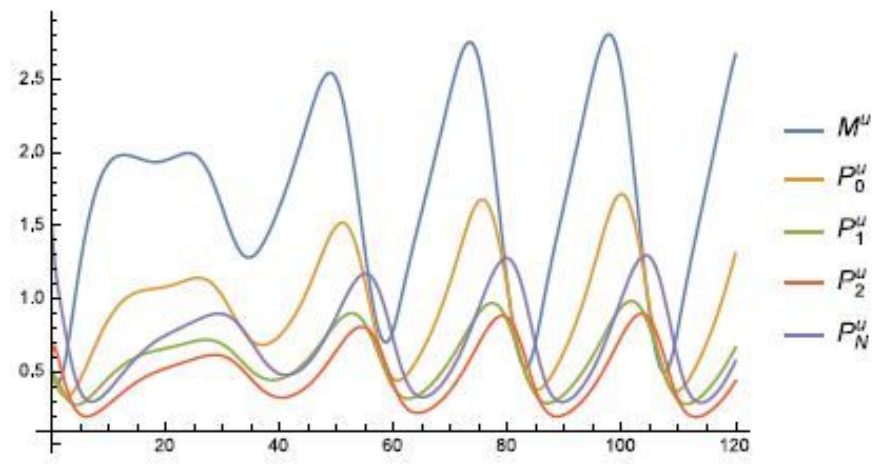

(c)

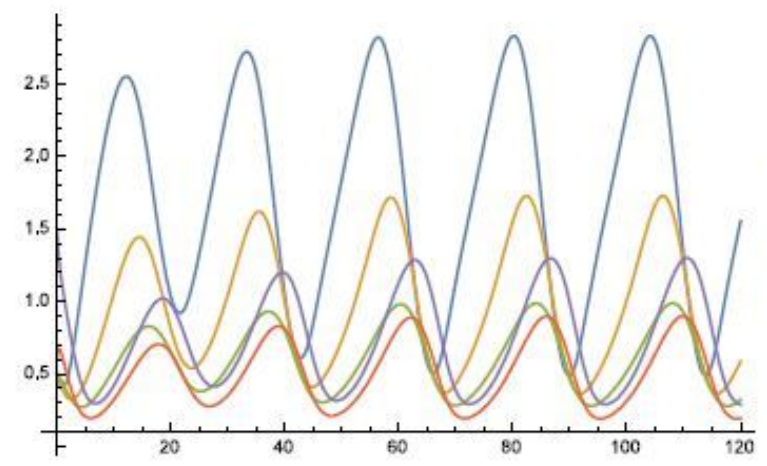

(e)

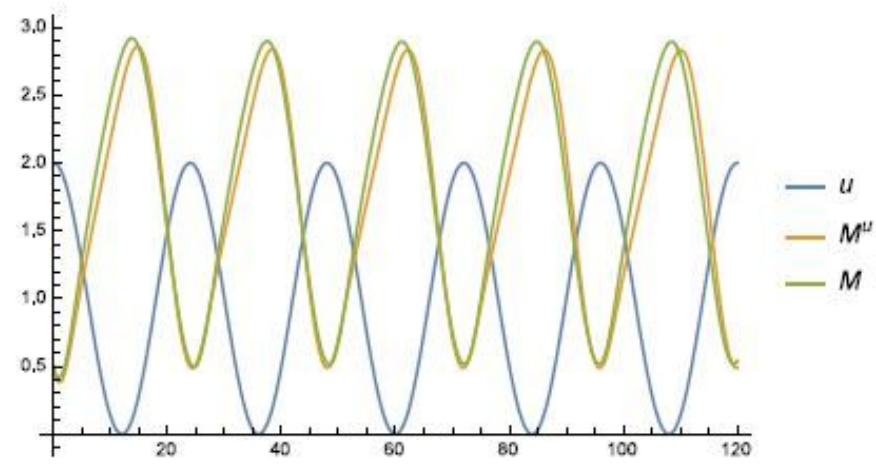

(b)

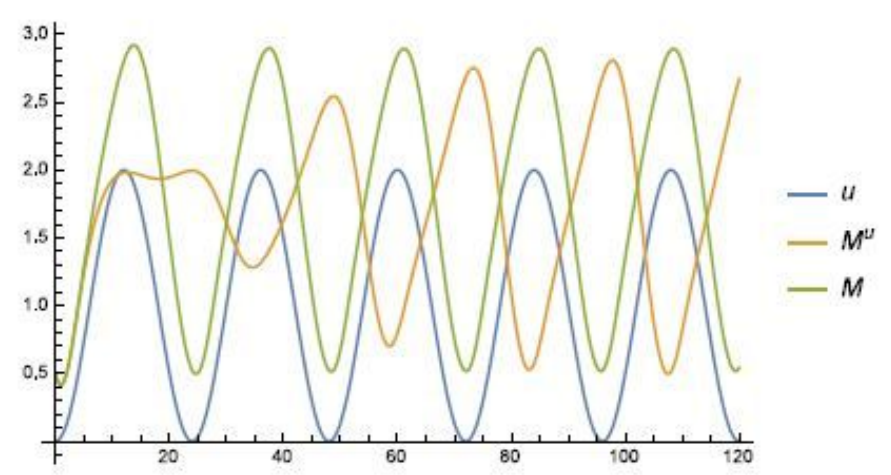

(d)

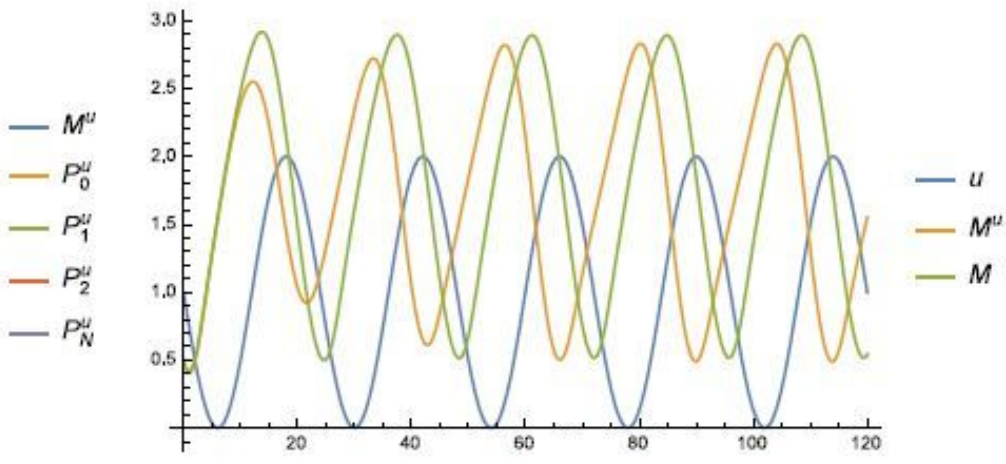

(f)

Figure 2

please see the manuscript file for the full caption 


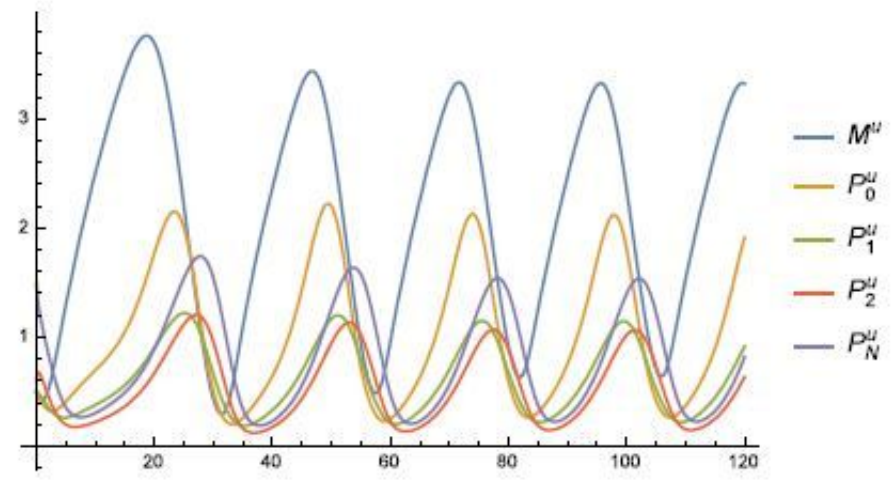

(a)

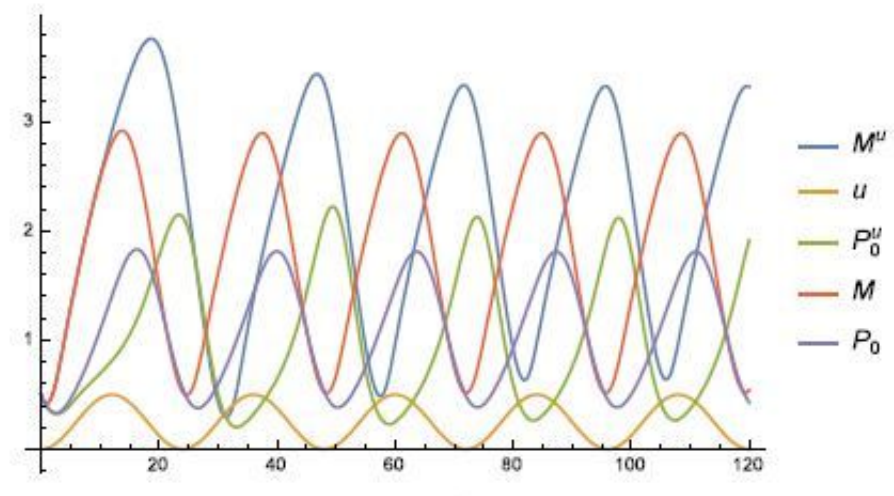

(b)

Figure 3

please see the manuscript file for the full caption 


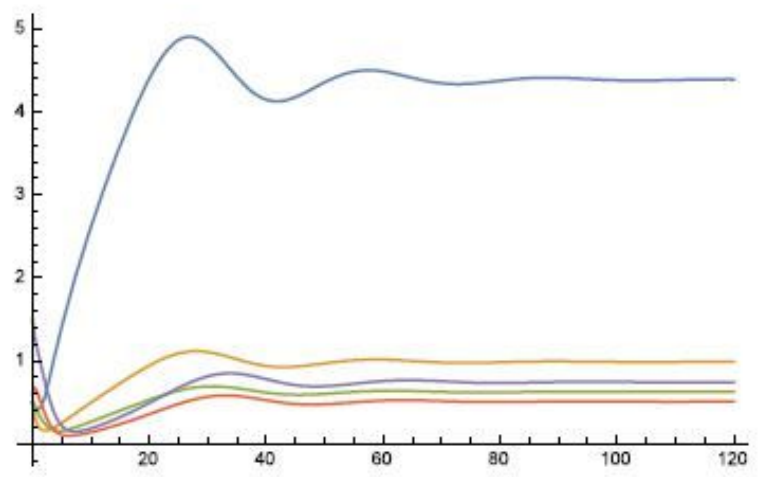

(a)

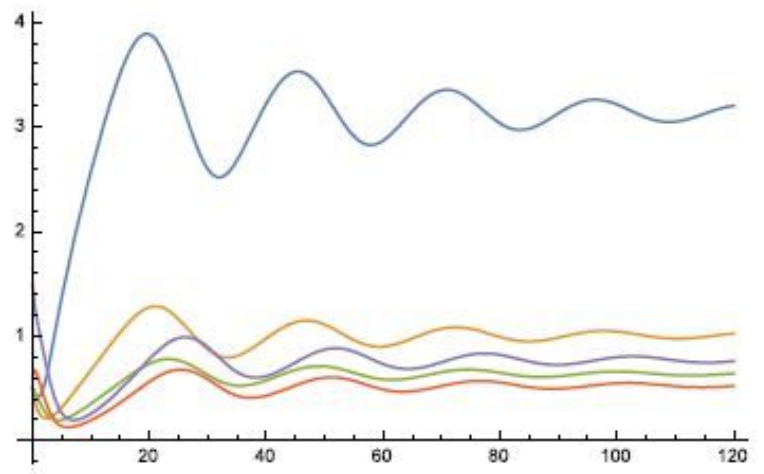

(c)

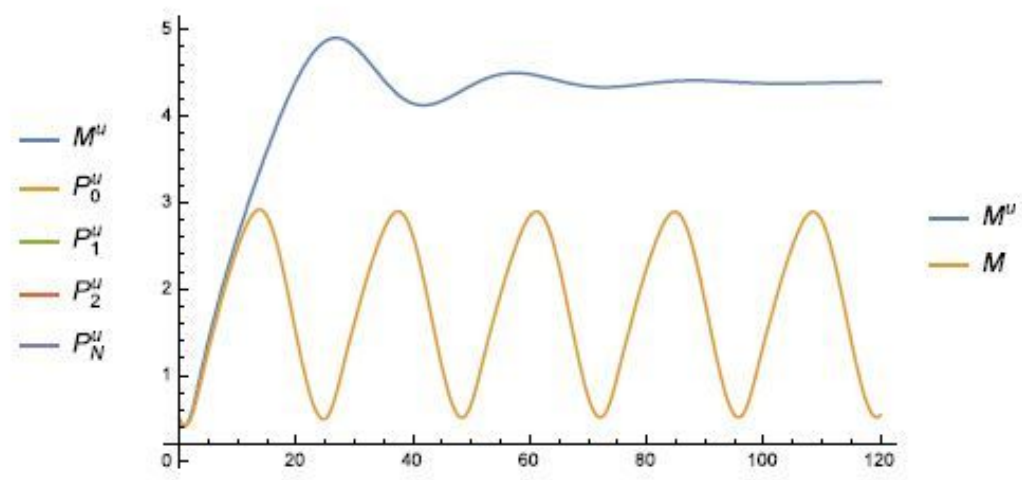

(b)

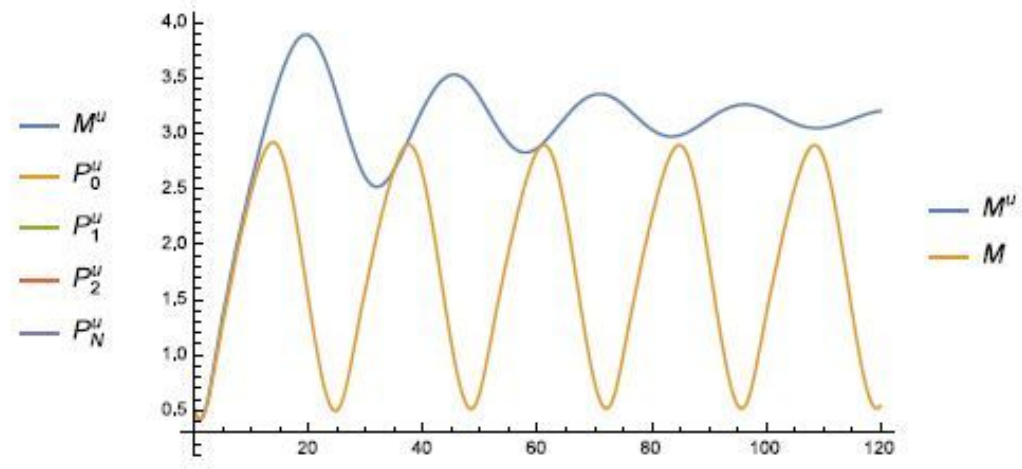

(d)

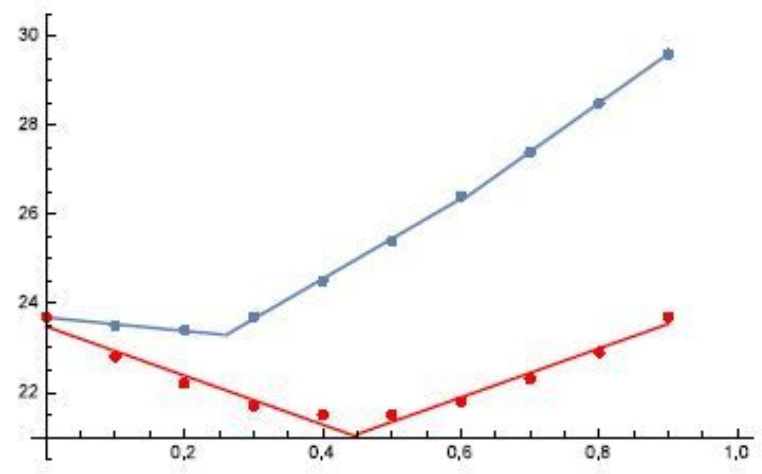

(e)

Figure 4

please see the manuscript file for the full caption 


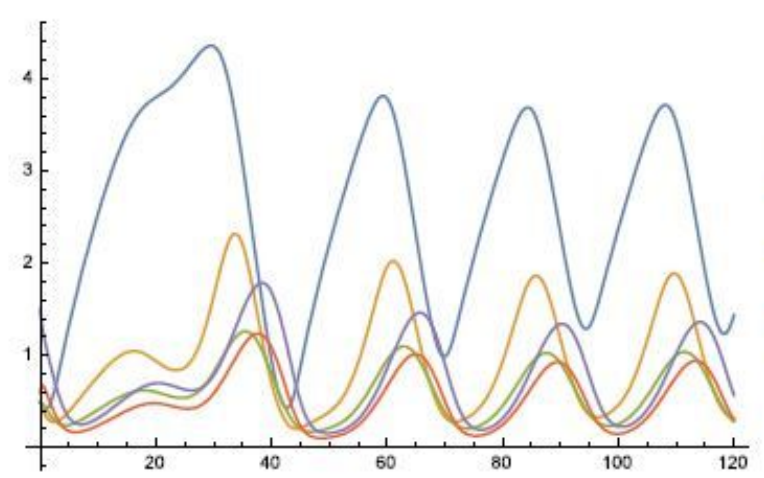

(a)

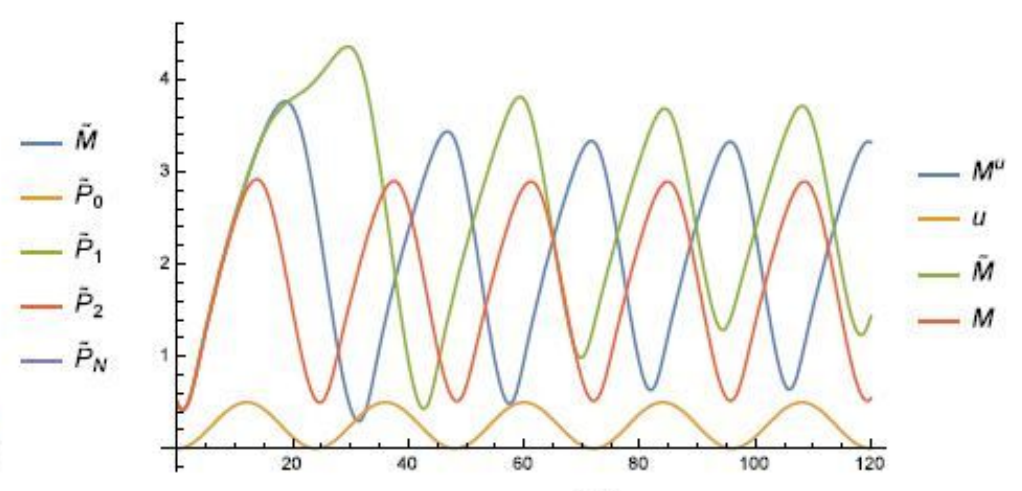

(b)

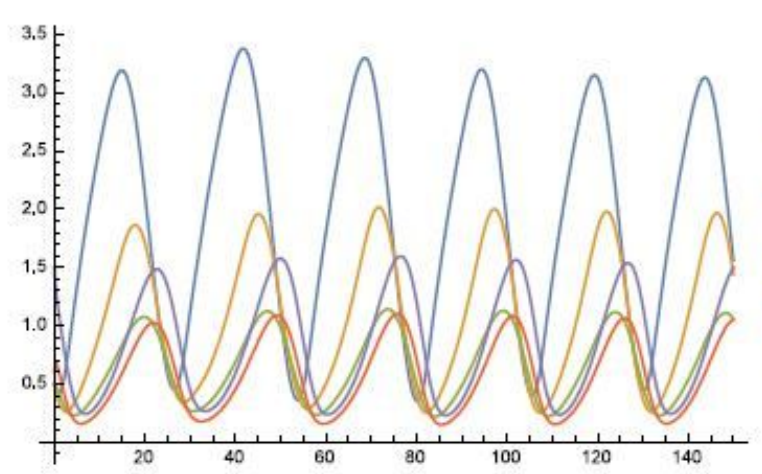

(c)

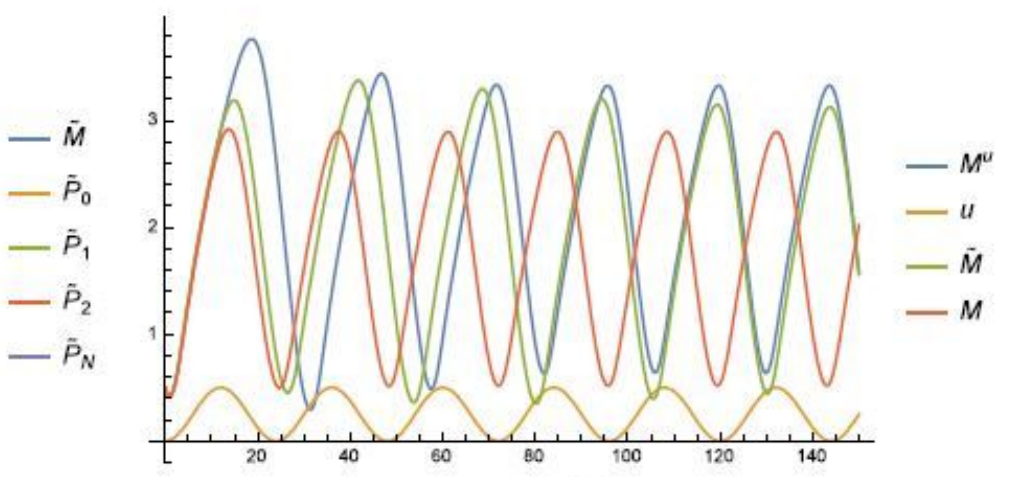

(d)

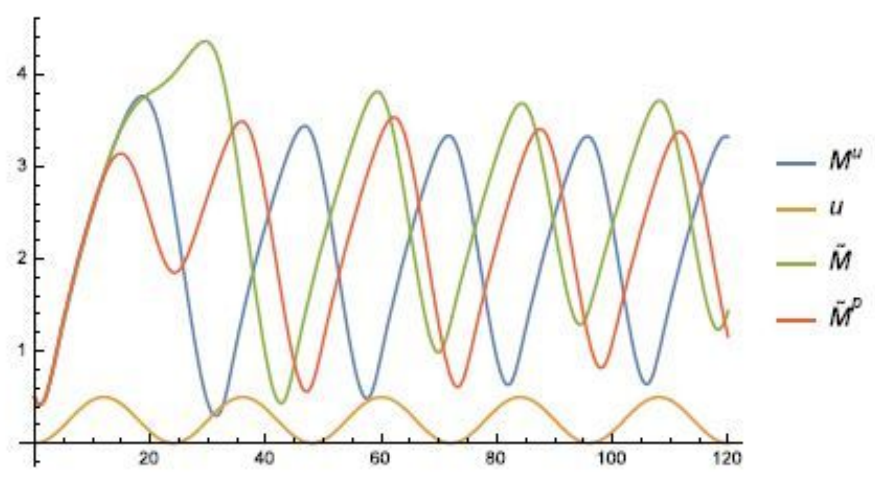

(e)

Figure 5

please see the manuscript file for the full caption 


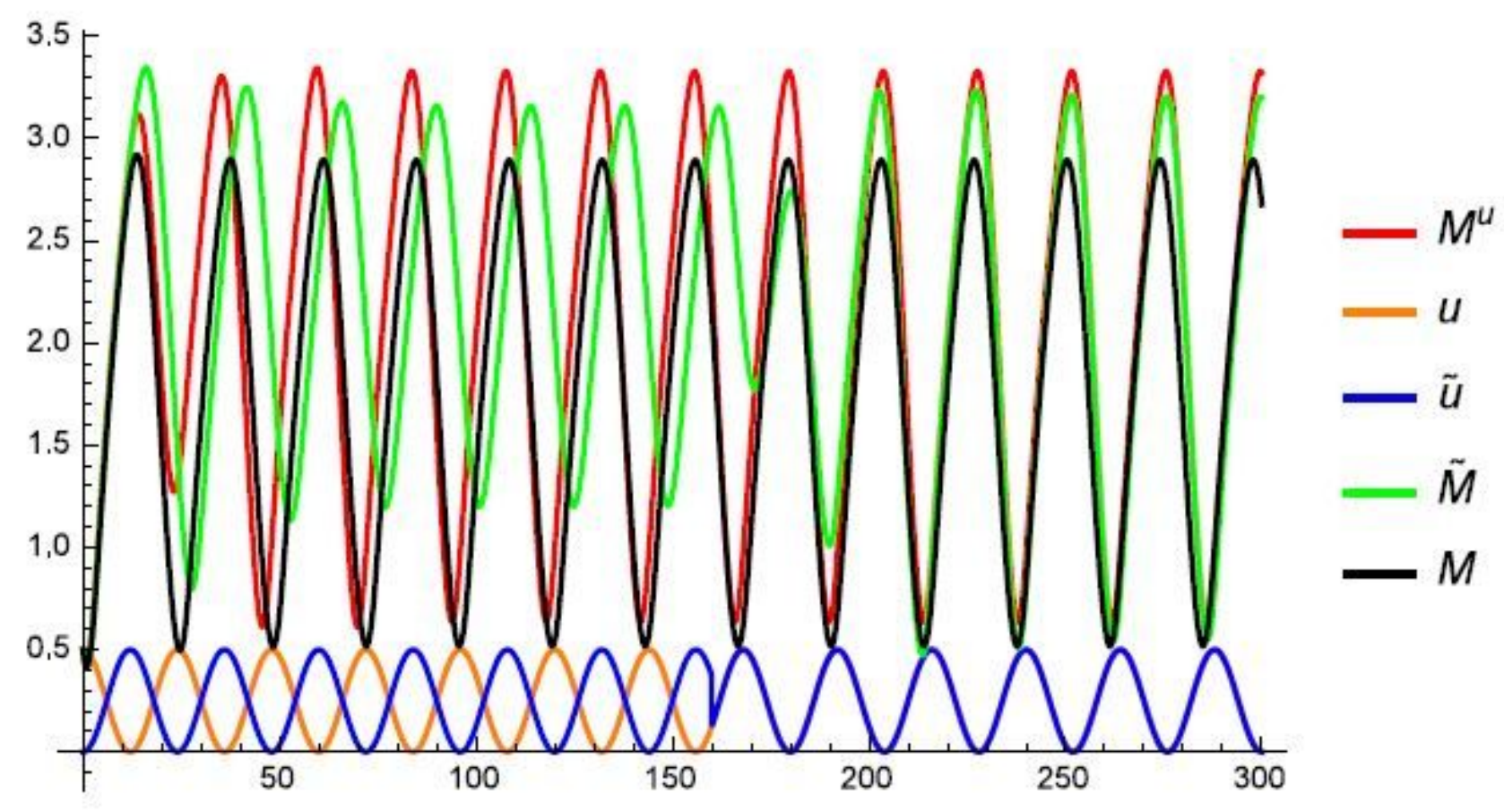

Figure 6

please see the manuscript file for the full caption

\section{Supplementary Files}

This is a list of supplementary files associated with this preprint. Click to download.

- SupplementaryMaterial.pdf 